\title{
Universally Verifiable Multiparty Computation from Threshold Homomorphic Cryptosystems
}

\author{
Berry Schoenmakers and Meilof Veeningen \\ Dept of Mathematics \& Computer Science \\ TU Eindhoven, The Netherlands \\ berry@win.tue.nl, m.veeningen@tue.nl
}

\begin{abstract}
Multiparty computation can be used for privacy-friendly outsourcing of computations on private inputs of multiple parties. A computation is outsourced to several computation parties; if not too many are corrupted (e.g., no more than half), then they cannot determine the inputs or produce an incorrect output. However, in many cases, these guarantees are not enough: we need correctness even if all computation parties may be corrupted; and we need that correctness can be verified even by parties that did not participate in the computation. Protocols satisfying these additional properties are called "universally verifiable". In this paper, we propose a new security model for universally verifiable multiparty computation, and we present a practical construction, based on a threshold homomorphic cryptosystem. We also develop a multiparty protocol for jointly producing non-interactive zero-knowledge proofs, which may be of independent interest.
\end{abstract}

\section{Introduction}

Multiparty computation (MPC) provides techniques for privacy-friendly outsourcing of computations. Intuitively, MPC aims to provide a cryptographic "black box" which receives private inputs from multiple "input parties"; performs a computation on these inputs; and provides the result to a "result party" (an input party, any third party, or the public). This black box is implemented by distributing the computation between multiple "computation parties", with privacy and correctness being guaranteed in case of passive corruptions (e.g., $\left[\mathrm{BCD}^{+} 09\right]$ ), active corruption of a minority of computation parties (e.g., [CDN01]), or active corruption of all-but-one computation parties (e.g., [DPSZ12]).

However, multiparty computation typically does not provide any guarantees in case all computation parties are corrupted. That is, the result party has to trust that at least some of the computation parties did their job, and has no way of independently verifying the result. In particular, the result party has no way of proving to an external party that his computation result is indeed correct. Universally verifiable multiparty computation addresses these issues by requiring that the correctness of the result can be verified by any party, even if all computation parties are corrupt [dH12]. It was originally introduced in the context of e-voting [CF85,SK95], but it is relevant whenever MPC is applied in a setting where not all of the parties that provide inputs or obtain outputs are 
participants in the computation. In particular, apart from contexts like e-voting where "the public" or an external watchdog wants to be sure of correctness, it is also useful in scenarios where (many) different input parties outsource a computation to the cloud and require a correctness guarantee.

Unfortunately, the state-of-the-art on universally verifiable MPC is unsatisfactory. The concept of universally verifiable MPC was first proposed in [dH12], where it was also suggested that it can be achieved for MPC based on threshold homomorphic cryptosystems. However, [dH12] does not provide a rigorous security model for universal verifiability or analysis of the proposed construction; and the construction has some technical disadvantages (e.g., a proof size depending on the number of computation parties). The scheme recently proposed in [BDO14] solves part of the problem. Their protocols provide "public auditability", meaning that anybody can verify the result of a computation, but only if that result is public. In particular, it is not possible for a result party to prove just that an encryption of the result is correct, which is important if this result is to be used in a later protocol without being revealed.

In this paper, we propose a new security model for universally verifiable multiparty computation, and a practical construction achieving it. As in [dH12], we adapt the well-known actively secure MPC protocols based on threshold homomorphic cryptosystems from [CDN01,DN03]. Essentially, these protocols perform computations on encrypted values; security against active adversaries is achieved by letting parties prove correctness of their actions using interactive zero-knowledge proofs. Such interactive proofs only convince parties present at the computation; but making them non-interactive makes them convincing also to external parties. Concretely, the result of a computation is a set of encryptions of the inputs, intermediate values, and outputs of the computation, along with non-interactive zero-knowledge proofs of their correctness. Correctness of the result depends just on the correct set-up of the cryptosystem. Privacy holds under the original conditions of [CDN01], i.e., if under half of the computation parties are corrupted; but as we discuss, this threshold can be raised to $n-$ 1 at the expense of sacrificing robustness. (Note that when computing with encryptions, we cannot hope to achieve privacy if all computation parties are corrupted: this would essentially require fully homomorphic encryption.)

We improve on [dH12] in two main ways. First, we provide a security model for universal verifiability (in the random oracle model), and security proofs for our protocols in that model. Second, we propose a new "multiparty" variant of the Fiat-Shamir heuristic to make the zero-knowledge proofs non-interactive, which may be of independent interest. Compared to [dH12], it eliminates the need for trapdoor commitments. Moreover, it makes the proof size independent of the number of parties performing the computation. We achieve this latter advantage by homomorphically combining contributions from the different parties.

As such, universally verifiable MPC provides a practical alternative to recent (single-party) techniques for verifiable outsourcing. Specifically, many papers on verifiable computation focus on efficient verification, but do not cover privacy [PHGR13,WB13]. Those works that do provide privacy, achieve this by combining costly primitives, e.g., fully homomorphic encryption with verifiable 


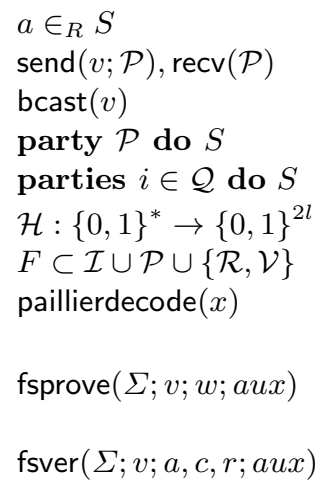

sample $a$ uniformly at random from $S$

send $v$ to/receive from $\mathcal{P}$ over secure channel exchange $v$ over broadcast channel let party $\mathcal{P}$ perform $S$; other parties do nothing let parties $i \in \mathcal{Q}$ perform $S$ in parallel

cryptographic hash function ( $l$ security parameter) global variable: set of parties found to misbehave threshold Paillier decoding (p. 6):

$$
((x-1) \div N)\left(4 \Delta^{2}\right)^{-1} \bmod N
$$

Fiat-Shamir proof (p. 8): $(a, s):=\Sigma \cdot \operatorname{ann}(v, w)$; $c:=\mathcal{H}(v\|a\| \mid a u x) ; r:=\Sigma \cdot \operatorname{res}(v, w, a, s, c) ; \pi:=(a, c, r)$ verification of Fiat-Shamir $\Sigma$-proof (p. 8):

$$
\mathcal{H}(v \||| \mid a u x)=c \wedge \Sigma \cdot \operatorname{ver}(v ; a ; c ; r)
$$

Fig. 1. Notation in algorithms, protocols, and processes

computation [FGP14]; or functional encryption with garbled circuits $\left[\mathrm{GKP}^{+} 13\right]$. A recent work $\left[\mathrm{ACG}^{+} 14\right]$ also considers the possibility of achieving verifiable computation with privacy by distributing the computation; but it does not guarantee correctness if all computation parties are corrupted, nor does it allow third parties to be convinced of this fact. In contrast, our methods guarantee correctness even if all computation parties are corrupted, and even convince other parties than the input party. In particular, any third party can be convinced, and the computation may involve the inputs of multiple mutually distrusting input parties. Moreover, in contrast to the above works, our methods rely on basic cryptographic primitives such as $\Sigma$-protocols and the threshold homomorphic Paillier cryptosystem, readily available nowadays in cryptographic libraries like SCAPI [EFLL12].

Outline First, we briefly recap the CDN scheme for secure computation in the presence of active adversaries from [CDN01,DN03], instantiated using Paillier encryption (Section 2). Then, we show how the proofs in this protocol can be made non-interactive using the Fiat-Shamir heuristic and our new multiparty variant (Section 3). Finally, we propose a security model for universally verifiable MPC, and show that CDN with non-interactive proofs is universally verifiable (Section 4). We conclude in Section 5. We list potentially non-obvious notation in our pseudocode in Figure 1.

\section{Secure Computation from Threshold Cryptography}

We review the "CDN protocol" [CDN01] for secure computation in the presence of active adversaries based on a threshold homomorphic cryptosystem. The protocol involves $m$ input parties $i \in \mathcal{I}, n$ computation parties $i \in \mathcal{P}$, and a result party $\mathcal{R}$. The aim of the protocol is to compute a function $f\left(x_{1}, \ldots, x_{m}\right)$ (seen as an arithmetic circuit) on private inputs $x_{i}$ of the input parties, such that the result party obtains the result. 


\subsection{Computation using a Threshold Homomorphic Cryptosystem}

The protocol uses a $(t, n)$-threshold homomorphic cryptosystem, with $t=\lceil n / 2\rceil$. In such a cryptosystem, anybody can encrypt a plaintext using the public key; add two ciphertexts to obtain a (uniquely determined) encryption of the sum of the corresponding plaintexts; and multiply a ciphertext by a constant to obtain a (uniquely determined) encryption of the product of the plaintext with the constant. Decryption is only possible if at least $t$ out of the $n$ decryption keys are known. A well-known homomorphic cryptosystem is the Paillier cryptosystem [Pai99]: here, the public key is an RSA modulus $N=p q ; a \in \mathbb{Z}_{N}$ is encrypted with randomness $r \in \mathbb{Z}_{N}^{*}$ as $(1+N)^{a} r^{N} \in \mathbb{Z}_{N^{2}}^{*}$; and the product of two ciphertexts is an encryption of the sum of the two corresponding plaintexts. (From now on, we suppress moduli for readability.) A threshold variant of this cryptosystem was presented in [DJ01]. The (threshold) decryption procedure is a bit involved; we postpone its discussion until Section 2.2. The CDN protocol can also be instantiated with other cryptosystems; but in this paper, we will focus on the Paillier instantiation.

Computation of $f\left(x_{1}, \ldots, x_{m}\right)$ is performed in three phases: the input phase, the computation phase, and the output phase. In the input phase, each input party encrypts its input $x_{i}$, and broadcasts the encryption $X_{i}$. In the computation phase, the function $f$ is evaluated gate-by-gate. Addition and subtraction are performed using the homomorphic property of the encryption scheme. For multiplication $^{1}$ of $X$ and $Y$, each computation party $i \in \mathcal{P}$ chooses a random value $d_{i}$, and broadcasts encryptions $D_{i}$ of $d_{i}$ and $E_{i}$ of $d_{i} \cdot y$. The computation parties then compute $X \cdot D_{1} \cdots D_{n}$, and threshold decrypt it to learn $x+d_{1}+\ldots+d_{n}$. Observe that this allows them to compute an encryption of $\left(x+d_{1}+\ldots+d_{n}\right) \cdot y$, and hence, using the $E_{i}$, also an encryption of $x \cdot y$. Finally, in the output phase, when the result of the computation has been computed as encryption $X$ of $x$, the result party obtains $x$ by broadcasting random encryption $D$ of $d$ and obtaining a threshold decryption $x-d$ of $X \cdot D^{-1}$.

Active security is achieved by letting the parties prove correctness of all information they exchange. Namely, the input parties prove knowledge of their inputs $X_{i}$ (this prevents parties from choosing inputs depending on other inputs). The computation parties prove knowledge of $D_{i}$, and prove that $E_{i}$ is indeed a correct multiplication of $D_{i}$ and $Y$; and they prove the correctness of their contributions to the threshold decryption of $X \cdot D_{1} \cdots D_{n}$ and $X \cdot D^{-1}$. Finally, the result party proves knowledge of $D$. We now discuss these proofs of correctness and their influence on the security of the overall protocol.

\subsection{Proving Correctness of Results}

The techniques in the CDN protocol for proving correctness are based on $\Sigma$ protocols. Recall that a $\Sigma$-protocol for a binary relation $R$ is a three-move protocol in which a potentially malicious prover convinces a honest verifier that he

\footnotetext{
1 Here, we use the improved multiplication protocol from [DN03]: the multiplication protocol from [CDN01] has a subtle problem, in which the subroutine for additively sharing an encrypted value requires unknown encryption randomness to be returned.
} 


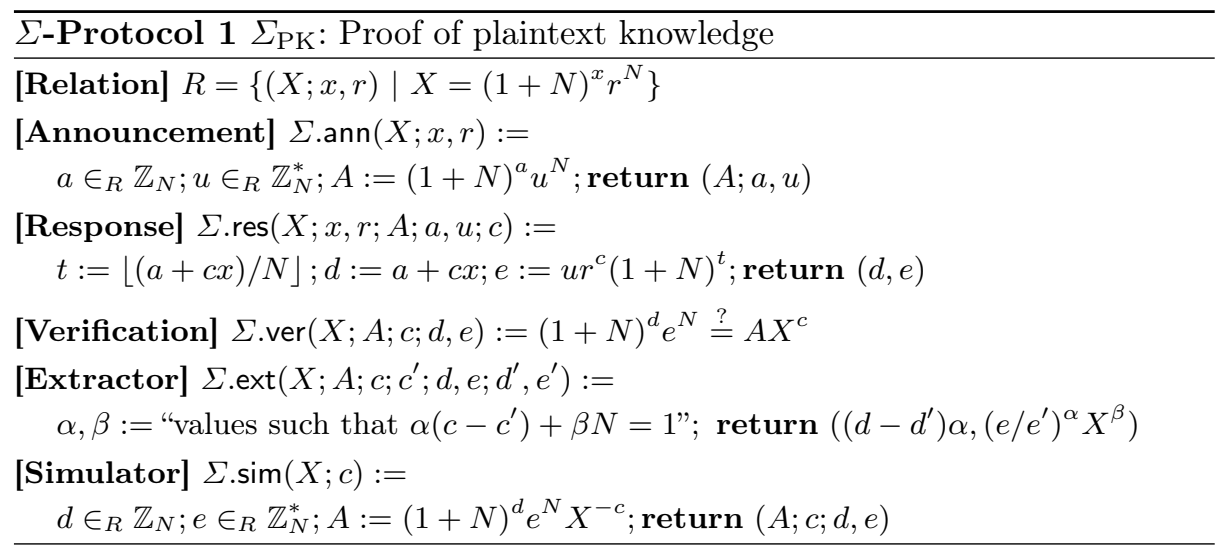

knows a witness $w$ for statement $v$ such that $(v ; w) \in R$. First, the prover sends an announcement (computed using algorithm $\Sigma$.ann) to the verifier; the verifier responds with a uniformly random challenge; and the prover sends his response (computed using algorithm $\Sigma$.res), which the verifier verifies (using predicate $\Sigma$.ver). $\Sigma$-protocols satisfy the following properties:

Definition 1. Let $R \subset V \times W$ be a binary relation and $L_{R}=\{v \in V \mid \exists w \in$ $W:(v ; w) \in R\}$ its language. Let $\Sigma$ be a collection of p.p.t. algorithms $\Sigma$.ann, $\Sigma$.res, $\Sigma$.sim, $\Sigma$.ext, and polynomial time predicate $\Sigma$.ver. Let $C$ be a finite set called the challenge space. Then $\Sigma$ is a $\Sigma$-protocol for relation $R$ if:

Completeness If $(a ; s) \leftarrow \Sigma \cdot \operatorname{ann}(v ; w), c \in C$, and $r \leftarrow \Sigma \cdot \operatorname{res}(v ; w ; a ; s ; c)$, then $\Sigma$.ver $(v ; a ; c ; r)$.

Special soundness If $v \in V, c \neq c^{\prime}, \Sigma \cdot \operatorname{ver}(v ; a ; c ; r)$, and $\Sigma \cdot \operatorname{ver}\left(v ; a ; c^{\prime} ; r^{\prime}\right)$, then $w \leftarrow \Sigma \cdot \operatorname{ext}\left(v ; a ; c ; c^{\prime} ; r ; r^{\prime}\right)$ satisfies $(v ; w) \in R$.

Special honest-verifier zero-knowledgeness If $v \in L_{R}, c \in C$, then $(a ; r) \leftarrow$ $\Sigma \cdot \operatorname{sim}(v ; c)$ has the same probability distribution as $(a ; r)$ obtained by $(a ; s) \leftarrow$ $\Sigma$.ann $(v ; w), r \leftarrow \Sigma \cdot \operatorname{res}(v ; w ; a ; s ; c)$. If $v \notin L_{R}$, then $(a ; r) \leftarrow \Sigma \cdot \operatorname{sim}(v ; c)$ satisfies $\Sigma . \operatorname{ver}(v ; a ; c ; r)$.

Completeness states that a protocol between a honest prover and verifier succeeds; special soundness states that there exists an extractor $\Sigma$.ext that can extract a witness from two conversations with the same announcement; and special honest-verifier zero-knowledgeness states that there exists a simulator $\Sigma$.sim that can generate conversations with the same distribution as full protocol runs without knowing the witness. While special honest-verifier zero-knowledgeness demands an identical distribution for the simulation, statistical indistinguishability is sufficient for our purposes; in this case, we speak of a "statistical $\Sigma$ protocol". In the remainder, we will need that our $\Sigma$-protocols have "non-trivial announcements", in the sense that when $(a ; r)$ and $\left(a^{\prime} ; r^{\prime}\right)$ are both obtained from $\Sigma \cdot \operatorname{sim}(v ; c)$, then with overwhelming probability, $a \neq a^{\prime}$. (Indeed, this will be the case for all $\Sigma$-protocols in this paper.) This property, which is required by the Fiat-Shamir heuristic [AABN08], essentially follows from the hardness of the relation; see Appendix B for details. 


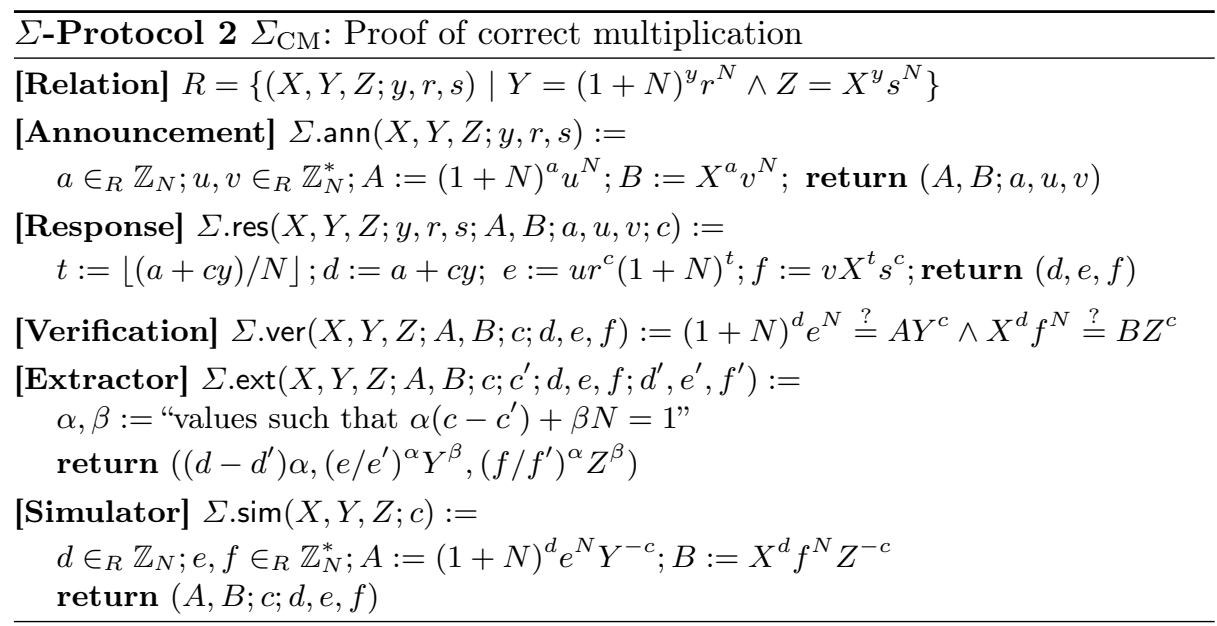

The CDN protocol uses a sub-protocol in which multiple parties simultaneously provide proofs based on the same challenge, called the "multiparty $\Sigma$ protocol". Namely, suppose each party from a set $P$ wants to prove knowledge of a witness for a statement $v_{i} \in L_{R}$ with some $\Sigma$-protocol. To achieve this, each party in $P$ broadcasts a commitment to its announcement; then, the computation parties jointly generate a challenge; and finally, all parties in $P$ broadcast their response to this challenge, along with an opening of their commitment. The multiparty $\Sigma$-protocol is used as a building block in the CDN protocol by constructing a simulator that provides proofs on behalf of honest parties without knowing their witnesses ("zero-knowledgeness"), and extracts witnesses from corrupted parties that give correct proofs ("soundness").

The CDN protocol uses three $\Sigma$-protocols: $\Sigma_{\mathrm{PK}}$ proving plaintext knowledge, $\Sigma_{\mathrm{CM}}$ proving correct multiplication, and $\Sigma_{\mathrm{CD}}$ proving correct decryption. The first two are due to [CDN01] (which also proves that they are $\Sigma$-protocols). $\Sigma_{\mathrm{PK}}$ ( $\Sigma$-Protocol 1) proves knowledge of $x, r$ such that $X=(1+N)^{x} r^{N}$ is an encryption of $x$ with randomness $r$. $\Sigma_{\mathrm{CM}}(\Sigma$-Protocol 2) proves knowledge of $(y, r, s)$ for $(X, Y, Z)$ such that $Y=(1+N)^{y} r^{N}$ is an encryption of $y$ with randomness $r$ and $Z=X^{y} s^{N}$ is an encryption of the product of the plaintexts of $X$ and $Y$ randomised with $s$.

Proof $\Sigma_{\mathrm{CD}}$ of correct decryption ( $\Sigma$-protocol 3 ) is due to [Jur03]. In the threshold variant of Paillier encryption due to Damgård and Jurik [DJ01,Jur03], safe primes $p=2 p^{\prime}+1, q=2 q^{\prime}+1$ are used for the RSA modulus $N=p q$. Key generation involves generating a secret value $d$ such that, given $c^{\prime}=c^{4 \Delta^{2}} d$, anybody can compute the plaintext of $c$ by "decoding" $c^{\prime}$ as paillierdecode $\left(c^{\prime}\right):=$ $\left(\left(c^{\prime}-1\right) \div N\right)\left(4 \Delta^{2}\right)^{-1} \bmod N$. Here, $\Delta=n !$ and $\div$ denotes division as integers (using $\left.N \mid c^{\prime}-1\right)$. The value $d$ is then $(t, n)$ Shamir-shared modulo $N p^{\prime} q^{\prime}$ between the computation parties as shares $s_{i}$. Threshold decryption is done by letting $t$ parties each compute $c_{i}=c^{2 \Delta s_{i}}$; the value $c^{4 \Delta^{2} d}$ is obtained by applying Shamir reconstruction "in the exponent". Correct decryption is proven with respect to a public set of verification values. Namely, the public key includes values $v$, 


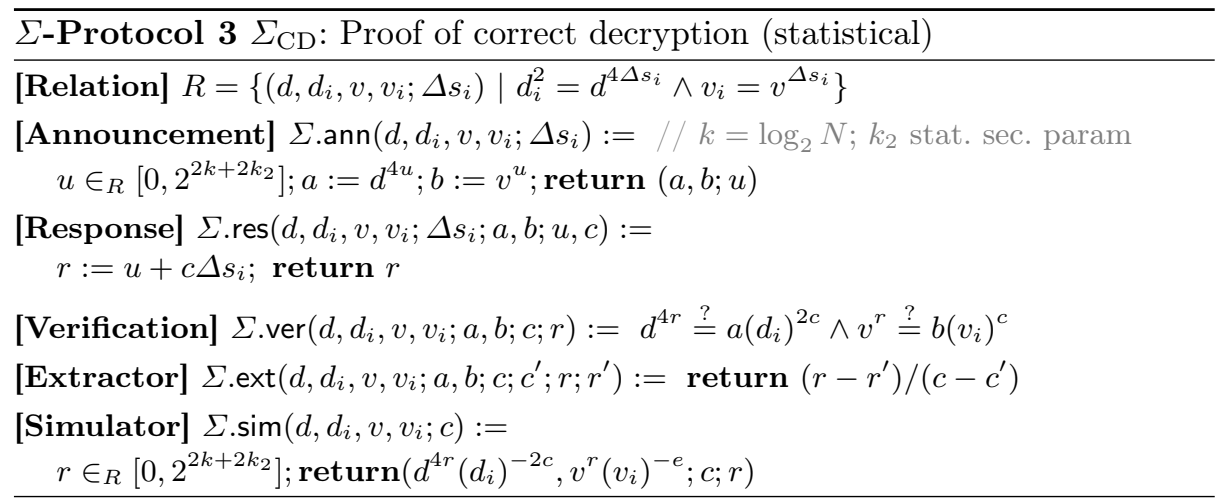

$v_{0}=v^{\Delta^{2} d}$, and $v_{i}=v^{\Delta s_{i}}$ for all computation parties $i \in \mathcal{P}$. Hence, in $\Sigma_{\mathrm{CD}}$, parties prove correctness of their decryption shares $c_{i}$ of $c$ by proving knowledge of $\Delta s_{i}=\log _{c^{4}}\left(c_{i}^{2}\right)=\log _{v}\left(v_{i}\right)$ for $\left(c, c_{i}, v, v_{i}\right)$. (In the same way, $v_{0}$ can be used to prove correctness of $c^{\prime}$ with respect to $c$ using a single instance of $\Sigma_{\mathrm{CD}}$.) Note that this is a statistical $\Sigma$-protocol: this is because witness $\Delta s_{i}$ is a value modulo the secret value $N p^{\prime} q^{\prime}$, so modulo reduction is not possible.

\subsection{Security of the CDN Protocol}

In [CDN01], it is shown that the CDN protocol implements secure function evaluation in Canetti's non-concurrent model [Can98] if only a minority of computation parties are corrupted. Essentially, this means that in this case, the computation succeeds; the result is correct; and the honest parties' inputs remain private. This conclusion is true assuming honest set-up and security of the Paillier encryption scheme and the trapdoor commitment scheme used. If a majority of computation parties is corrupted, then because threshold $\lceil n / 2\rceil$ is used for the threshold cryptosystem, privacy is broken. As noted [ST06,IPS09], this can be remedied by raising the threshold, but in that case, the corrupted parties can make the computation break down at any point by refusing to cooperate. In Section 4.1, we present a variant of this model in which we prove the security of our protocols (using random oracles but no trapdoor commitments).

\section{Multiparty Non-Interactive Proofs}

In this section, we show how to produce non-interactive zero-knowledge proofs in a multiparty way. At several points in the above CDN protocol, all parties from a set $P$ prove knowledge of witnesses for certain statements; the computation parties are convinced that those parties that succeed, do indeed know a witness. In $\mathrm{CDN}$, these proofs are interactive; but for universal verifiability, we need noninteractive proofs that convince any third party. The traditional method to make proofs non-interactive is the Fiat-Shamir heuristic; in Section 3.1, we outline it, and show that it is problematic in a multiparty setting. In Section 3.2, we present 
a new, "multiparty" Fiat-Shamir heuristic that works in our setting, and has the advantage of achieving smaller proofs by "homomorphically combining" the proofs of individual parties. In the remainder, $C \subset \mathcal{I} \cup \mathcal{P} \cup\{\mathcal{R}, \mathcal{V}\}$ denotes the set of corrupted parties; and $F$ denotes the set of parties who failed to provide a correct proof when needed; this only happens for corrupted parties, so $F \subset C$.

Our results are in the random oracle model [BR93,Wee09], an idealised model of hash functions. In this model, evaluations of the hash function $\mathcal{H}$ are modelled as queries to a "random oracle" $\mathcal{O}$ that evaluates a perfectly random function. When simulating an adversary, a simulator can intercept these oracle queries and answer them at will, as long as the answers look random to the adversary. Security in the random oracle model does not generally imply security in the standard model [GK03], but it is often used because it typically gives simple, efficient protocols, and its use does not seem to lead to security problems in practice [Wee09]. See Appendix A for a detailed description of our use of random oracles; and Section 5 for a discussion of the real-world implications of the particular flavour of random oracles we use.

\subsection{The Fiat-Shamir Heuristic and Witness-Extended Emulation}

The obvious way of making the proofs in the CDN protocol non-interactive, is to apply the Fiat-Shamir heuristic to all individual $\Sigma$-protocols. That is, party $i \in P$ produces proof of knowledge $\pi$ of a witness for statement $v$ as follows ${ }^{2}$ :

$$
(a ; s):=\Sigma \cdot \operatorname{ann}(v ; w) ; c:=\mathcal{H}(v\|a\| a u x) ; r:=\Sigma \cdot \operatorname{res}(v ; w ; a ; s ; c) ; \pi:=(a ; c ; r) .
$$

Let us denote this procedure fsprove $(\Sigma ; v ; w ; a u x)$. A verifier accepts those proofs $\pi=(a ; c ; r)$ for which $\operatorname{fsver}(\Sigma ; v ; \pi ;$ aux $)$ holds, where fsver $(\Sigma ; v ; a, c, r ; a u x)$ is defined as $\mathcal{H}(v\|a\| a u x)=c \wedge \Sigma \cdot \operatorname{ver}(v ; a ; c ; r)$.

Recall that security proofs require a simulator that simulates proofs of honest parties (zero-knowledgeness) and extracts witnesses of corrupted parties (soundness). In the random oracle model, Fiat-Shamir proofs for honest parties can be simulated by simulating a $\Sigma$-protocol conversation $(a, c, r)$ and programming the random oracle so that $\mathcal{H}(v\|a\| a u x)=c$. Witnesses of corrupted parties can be extracted by rewinding the adversary to the point where it made an oracle query for $v\|a\| a u x$ and supplying a different value; but, as we discuss in Appendix A, this extraction can make the simulator very inefficient. In fact, if Fiat-Shamir proofs take place in $R$ different rounds, then extracting witnesses may increase the running time of the simulator by a factor $O(R !)$. The reason is that the oracle query for a proof in one round may have in fact already been made in a previous round, in which case rewinding the adversary to extract one witness requires recursively extracting witnesses for all intermediate rounds. Hence, we can essentially only use the Fiat-Shamir heuristic in a constant number of rounds.

\footnotetext{
${ }^{2}$ Here, aux should contain at least the prover's identity. Otherwise, corrupted parties could replay proofs by honest parties, which breaks the soundness property below because witnesses for these proofs cannot be extracted by rewinding the adversary to the point of the oracle query and reprogramming the random oracle.
} 
Moreover, in the CDN protocol, applying the Fiat-Shamir heuristic to each individual proof has the disadvantage that the verifier needs to check a number of proofs that depends linearly on the number of computation parties. In particular, for each multiplication gate, the verifier needs to check $n$ proofs of correct multiplication and $t$ proofs of correct decryption. Next, we show that we can avoid both the technical problems with witness extended emulation and the dependence on the number of computation parties by letting the computation parties collaboratively produce "combined proofs". (As discussed in Appendix A, there are other ways of just solving the technical problems with witness extended emulation, but they are not easier than the method we propose.)

\subsection{Combined Proofs with the Multiparty Fiat-Shamir Heuristic}

The crucial observation (e.g., [Des93,KMR12]) allowing parties to produce noninteractive zero-knowledge proofs collaboratively is that, for many $\Sigma$-protocols, conversations of proofs with the same challenge can be "homomorphically combined". For instance, consider the classical $\Sigma$-protocol for proving knowledge of a discrete logarithm due to Schnorr [Sch89]. Suppose we have two Schnorr conversations proving knowledge of $x_{1}=\log _{g} h_{1}, x_{2}=\log _{g} h_{2}$, i.e., two tuples $\left(a_{1} ; c ; r_{1}\right)$ and $\left(a_{2} ; c ; r_{2}\right)$ such that $g^{r_{1}}=a_{1}\left(h_{1}\right)^{c}$ and $g^{r_{2}}=a_{2}\left(h_{2}\right)^{c}$. Then $g^{r_{1}+r_{2}}=\left(a_{1} a_{2}\right)\left(h_{1} h_{2}\right)^{c}$, so $\left(a_{1} a_{2} ; c ; r_{1}+r_{2}\right)$ is a Schnorr conversation proving knowledge of discrete logarithm $x_{1}+x_{2}=\log _{g}\left(h_{1} h_{2}\right)$. For our purposes, we demand that such homomorphisms satisfy two properties. First, when conversations of at least $\lceil n / 2\rceil$ parties are combined, the result is a valid conversation (the requirement of having at least $\lceil n / 2\rceil$ conversations is needed for decryption proofs to ensure that there are enough decryption shares). Second, when fewer than $\lceil n / 2\rceil$ parties are corrupted, the combination of different honest announcements with the same corrupted announcements is likely to lead to a different combined announcement. This helps to eliminate the rewinding problems for Fiat-Shamir discussed above.

Definition 2. Let $\Sigma$ be a $\Sigma$-protocol for relation $R \subset V \times W$. Let $\Phi$ be a collection of partial functions $\Phi$.stmt, $\Phi$.ann, and $\Phi$.resp. We call $\Phi$ a homomorphism of $\Sigma$ if:

Combination Let $c$ be a challenge; I a set of parties such that $|I| \geq\lceil n / 2\rceil$; and $\left\{\left(v_{i} ; a_{i} ; r_{i}\right)\right\}_{i \in I}$ a collection of statements, announcements, and responses. If $\Phi . \operatorname{stmt}\left(\left\{v_{i}\right\}_{i \in I}\right)$ is defined and for all $i, \Sigma$.ver $\left(v_{i} ; a_{i} ; c ; r_{i}\right)$ holds, then also $\Sigma . \operatorname{ver}\left(\Phi \cdot \operatorname{stmt}\left(\left\{v_{i}\right\}_{i \in I}\right) ; \Phi \cdot \operatorname{ann}\left(\left\{a_{i}\right\}_{i \in I}\right) ; c ; \Phi \cdot \operatorname{resp}\left(\left\{r_{i}\right\}_{i \in I}\right)\right)$.

Randomness Let $c$ be a challenge; $C \subset I$ sets of parties such that $|C|<$ $\lceil n / 2\rceil \leq|I| ;\left\{v_{i}\right\}_{i \in I}$ statements s.t. $\Phi . \operatorname{stmt}\left(\left\{v_{i}\right\}_{i \in I}\right)$ is defined; and $\left\{a_{i}\right\}_{i \in I \cap C}$ announcements. If $\left(a_{i} ; \cdot\right),\left(a_{i}^{\prime} ; \cdot\right) \leftarrow \Sigma \cdot \operatorname{sim}\left(v_{i} ; c\right) \forall i \in I \backslash C$, then with overwhelming probability, $\Phi$.ann $\left(\left\{a_{i}\right\}_{i \in I}\right) \neq \Phi$.ann $\left(\left\{a_{i}\right\}_{i \in I \cap C} \cup\left\{a_{i}^{\prime}\right\}_{i \in I \backslash C}\right)$.

Given a $\Sigma$-protocol with homomorphism $\Phi$, parties holding witnesses $\left\{w_{i}\right\}$ for statements $\left\{v_{i}\right\}$ can together generate a Fiat-Shamir proof $(a ; \mathcal{H}(v\|a\| a u x) ; r)$ of knowledge of a witness for the "combined statement" $v=\Phi \cdot \operatorname{stmt}\left(\left\{v_{i}\right\}\right)$. Namely, the parties each provide announcement $a_{i}$ for their own witness; compute $a=$ 


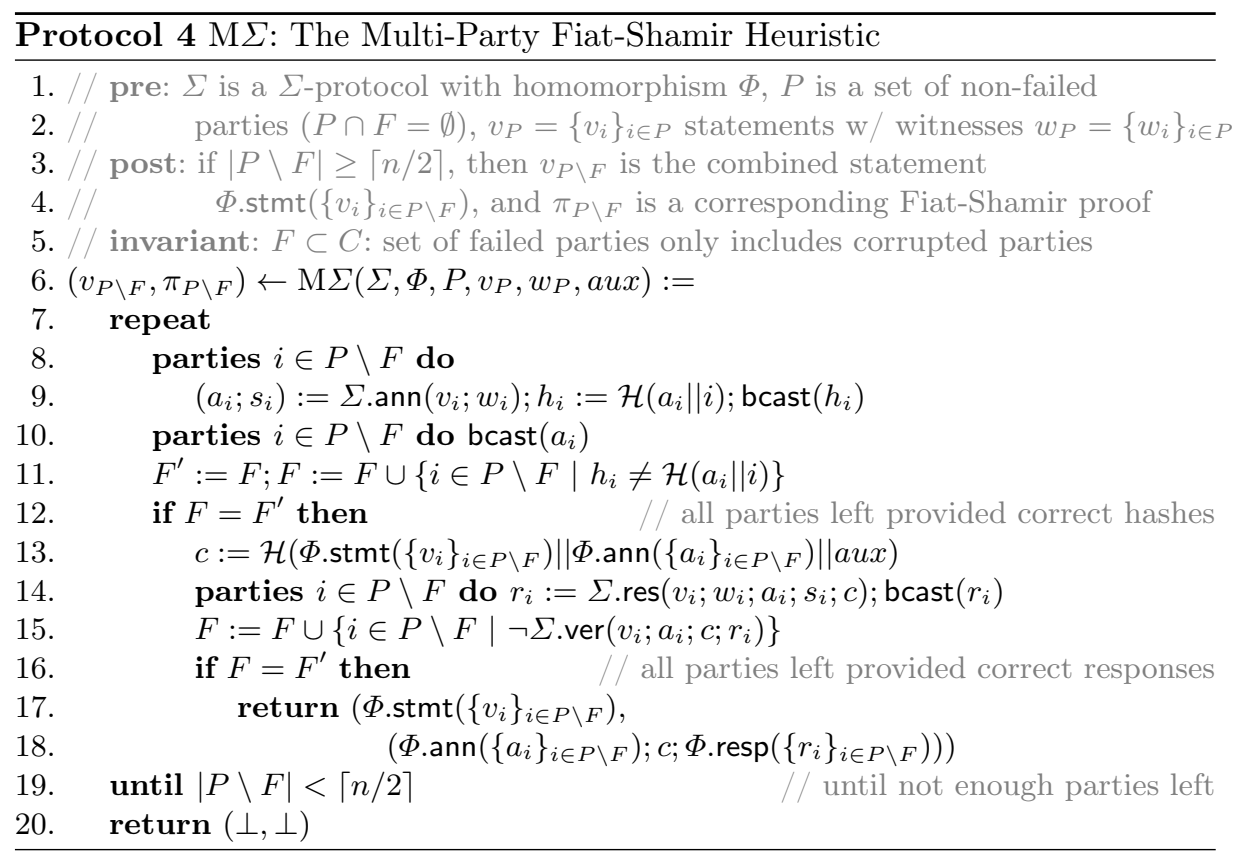

$\Phi$.ann $\left(\left\{a_{i}\right\}\right)$ and $\mathcal{H}(v\|a\| a u x)$; and provide responses $r_{i}$. Taking $r=\Phi . \operatorname{resp}\left(\left\{r_{i}\right\}\right)$, the combination property from the above definition guarantees that we indeed get a validating proof. However, we cannot simply let the parties broadcast their announcements in turn, because to prove security in that case, the simulator needs to provide the announcements for the honest parties without knowing the announcements of the corrupted parties, hence without being able to program the random oracle on the combined announcement. We solve this by starting with a round in which each party commits to its announcement (the same trick was used in a different setting in [NKDM03] $)^{3}$.

The multiparty Fiat-Shamir heuristic (Protocol 4) let parties collaboratively produce Fiat-Shamir proofs based on the above ideas. Apart from the above procedure (lines 8, 9, 10,13, and 14), the protocol also contains error handling. Namely, we throw out parties that provide incorrect hashes to their announcements (line 11) or incorrect responses (line 15). If we have correct responses for all correctly hashed announcements, then we apply the homomorphism (line 17-18); otherwise, we try again with the remaining parties. If the number of parties drops below $\lceil n / 2\rceil$, the homomorphism can no longer be applied, so we return with an error (line 20). Note that, as in the normal Fiat-Shamir heuristic, the announcements do not need to be stored if they can be computed from the challenge and response (as will be the case for the $\Sigma$-protocols we consider).

Concerning security, recall that we need a simulator that simulates proofs of honest parties without their witnesses (zero-knowledgeness) and extracts the

\footnotetext{
${ }^{3}$ As in [NKDM03], it may be possible to remove the additional round under the non-standard known-target discrete log problem.
} 
witnesses of corrupted parties (soundness). In Appendix B, we present such a simulator. Essentially, it "guesses" the announcements of the corrupted parties based on the provided hashes; then simulates the $\Sigma$-protocol for the honest parties; and programs the random oracle on the combined announcement. It obtains witnesses for the corrupted parties by rewinding to just before the honest parties provide their announcements: this way, the corrupted parties are forced to use the announcements that they provided the hashes of (hence special soundness can be invoked), whereas the honest parties can provide new simulated announcements by reprogramming the random oracle. The simulator requires that fewer than $\lceil n / 2\rceil$ provers are corrupted so that we can use the randomness property of the $\Sigma$-protocol homomorphism (Definition 2). (When more than $\lceil n / 2\rceil$ provers are corrupted, we use an alternative proof strategy that uses witness-extended emulation instead of this simulator.)

\subsection{Homomorphisms for the CDN Protocol}

In the CDN protocol, the multiparty Fiat-Shamir heuristic allows us to obtain a proof that multiplication was done correctly that is independent of the number of computation parties. Recall that, for multiplication of encryptions $X$ of $x$ and $Y$ of $y$, each computation party provides encryptions $D_{i}$ of $d_{i}$ and $E_{i}$ of $d_{i} \cdot y$, and proves that $E_{i}$ encrypts the product of the plaintexts of $Y$ and $D_{i}$; and each computation party provides decryption share $S_{i}$ of encryption $X D_{1} \cdots D_{n}$, and proves it correct. As we will show now, the multiplication proofs can be combined with homomorphism $\Phi_{\mathrm{CM}}$ into one proof that $\prod E_{i}$ encrypts the product of the plaintexts of $Y$ and $\prod D_{i}$; and the decryption proofs can be combined with homomorphism $\Phi_{\mathrm{CD}}$ into one proof that a combination $S_{0}$ of the decryption shares is correct. In the CDN protocol, the individual $D_{i}, E_{i}$, and $S_{i}$ are not relevant, so also the combined values convince a verifier of correct multiplication.

In more detail, the homomorphism $\Phi_{\mathrm{CM}}$ for $\Sigma_{\mathrm{CM}}$ is defined on statements $\left\{\left(X, Y_{i}, Z_{i}\right)\right\}_{i \in I}$ which share encryption $X$, and it proves that the multiplication on plaintexts of $X$ with $\prod Y_{i}$ is equal to $\prod Z_{i}$. We let $\Phi \cdot \operatorname{stmt}\left(\left\{\left(X, Y_{i}, Z_{i}\right)\right\}_{i \in I}\right)=$ $\left(X, \prod_{i \in I} Y_{i}, \prod_{i \in I} Z_{i}\right)$ and $\Phi \cdot \operatorname{ann}\left(\left\{A_{i}, B_{i}\right\}_{i \in I}\right)=\left(\prod_{i \in I} A_{i}, \prod_{i \in I} B_{i}\right)$. For the response, we would like to define $d=\sum_{i \in I} d_{i}, e=\prod_{i \in I} e_{i}$, and $f=\prod_{i \in I} f_{i}$; but because $\sum_{i \in I} d_{i}$ is computed modulo $N$, we need to add correction factors to $e$ and $f: e=\left(\prod_{i \in I} e_{i}\right)(1+N)^{k}$ and $f=\left(\prod_{i \in I} f_{i}\right) Y^{k}$ (where $\left.k=\left\lfloor\left(\sum_{i \in I} d_{i}\right) / N\right\rfloor\right)$.

The homomorphism $\Phi_{\mathrm{CD}}$ for $\Sigma_{\mathrm{CD}}$ combines correctness proofs of decryption shares into a proof of correct decryption with respect to an overall verification value. Let $I \geq\lceil n / 2\rceil$ be sufficiently many parties to decrypt a ciphertext, let $\left\{\lambda_{i}\right\}_{i \in I}$ be Lagrange interpolation coefficients for these parties. (Note that $\lambda_{i}$ are not always integral; but we will always use $\Delta \lambda_{i}$, which are integral.) Let $s_{i}$ be their shares of the decryption key $d=\sum_{i \in I} \Delta \lambda_{i} s_{i}$. Recall that decryption works by letting each party $i \in I$ provide decryption share $c_{i}=c^{2 \Delta s_{i}}$; computing $c^{\prime}=$ $\prod_{i \in I} c_{i}^{2 \Delta \lambda_{i}} ;$ and from this determining the plaintext as paillierdecode $\left(c^{\prime}\right)$. Parties prove correctness of their decryption shares $c_{i}$ by proving that $\log _{c^{4}} c_{i}^{2}=\log _{v} v_{i}$, where $v, v_{i}$ are publicly known verification values such that $v_{i}=v^{\Delta s_{i}}$. Now, if 
$\log _{c^{4}} c_{i}^{2}=\log _{v} v_{i}$ for all $i$, then

$$
\log _{c^{4}} c^{\prime}=\log _{c^{4}} \prod_{i \in I} c_{i}^{2 \Delta \lambda_{i}}=\log _{v} \prod_{i \in I} v_{i}^{\Delta \lambda_{i}}=\log _{v} \prod_{i \in I}\left(v^{\Delta s_{i}}\right)^{\Delta \lambda_{i}}=\log _{v} v^{\Delta^{2} d} .
$$

Hence, decryption proofs for shares $c_{i}$ with respect to verification values $v_{i}$ can be combined into a decryption proof for $c^{\prime}$ with respect to verification value $v_{0}:=v^{\Delta^{2} d}$. Formally, $\Phi \cdot \operatorname{stmt}\left(\left\{\left(d, d_{i}, v, v_{i}\right)\right\}_{i \in I}=\left(d, \prod_{i \in I} c_{i}^{\Delta \lambda_{i}}, v, \prod_{i \in I} v_{i}^{\Delta \lambda_{i}}\right) ;\right.$ $\Phi \cdot \operatorname{ann}\left(\left\{\left(a_{i}, b_{i}\right)\right\}_{i \in I}\right)=\left(\prod_{i \in I} a_{i}^{\Delta \lambda_{i}}, \prod_{i \in I} b_{i}^{\Delta \lambda_{i}}\right) ;$ and $\Phi \cdot \operatorname{resp}\left(\left\{r_{i}\right\}_{i \in I}\right)=\sum \Delta \lambda_{i} r_{i}$. For the combination property of Definition 2, note that we really need $I \geq\lceil n / 2\rceil$ in order to apply Lagrange interpolation. For the randomness property, note that if $|C|<\lceil n / 2\rceil$, then at least one party in $I \notin C$ has a non-zero interpolation coefficient, hence the contribution of this party to the announcement ensures that the two combined announcements are different.

\section{Universally Verifiable MPC}

In the previous section, we have shown how to produce non-interactive zeroknowledge proofs in a multiparty way. We now use this observation to obtain universally verifiable MPC. We first define security for universally verifiable MPC; and then obtain universally verifiable MPC by adapting the CDN protocol.

\subsection{Security Model for Verifiable MPC}

Our security model is an adaptation of the model of [Can98,CDN01] to the setting of universal verifiability in the random oracle model. We first explain the general execution model, which is as in [Can98,CDN01] but with a random oracle added; we then explain how to model verifiability in this execution model as the behaviour of the ideal-world trusted party. The general execution model compares protocol executions in the real and ideal world.

In the real world, a protocol $\pi$ between $m$ input parties $i \in \mathcal{I}, n$ computation parties $i \in \mathcal{P}$, a result party $\mathcal{R}$ and a verifier $\mathcal{V}$ is executed on an open broadcast network with rushing in the presence of an active static adversary $\mathcal{A}$ corrupting parties $C \subset \mathcal{I} \cup \mathcal{P} \cup\{\mathcal{R}, \mathcal{V}\}$. The protocol execution starts by incorruptibly setting up the Paillier threshold cryptosystem, i.e., generating public key pk = $\left(N, v, v_{0},\left\{v_{i}\right\}_{i \in \mathcal{P}}\right)$ with RSA modulus $N$ and verification values $v, v_{0}, v_{i}$, and secret key shares $\left\{s_{i}\right\}_{i \in \mathcal{P}}$ (see Section 2.2). Each input party $i \in \mathcal{I}$ gets input (pk, $x_{i}$ ); each computation party $i \in \mathcal{P}$ gets input (pk, $s_{i}$ ); and the result party $\mathcal{R}$ gets input pk. The adversary gets the inputs (pk, $\left\{x_{i}\right\}_{i \in \mathcal{I} \cap C},\left\{s_{i}\right\}_{i \in \mathcal{P} \cap C}$ ) of the corrupted parties, and has an auxiliary input $a$. During the protocol, parties can query the random oracle; the oracle answers new queries randomly, and repeated queries consistently. At the end of the protocol, each honest party outputs a value according to the protocol; the corrupted parties output $\perp$; and the adversary outputs a value at will. Define $\operatorname{EXEC}_{\pi, \mathcal{A}}\left(k,\left(x_{1}, \ldots, x_{m}\right), C, a\right)$ to be the random variable, given security parameter $k$, consisting of the outputs 


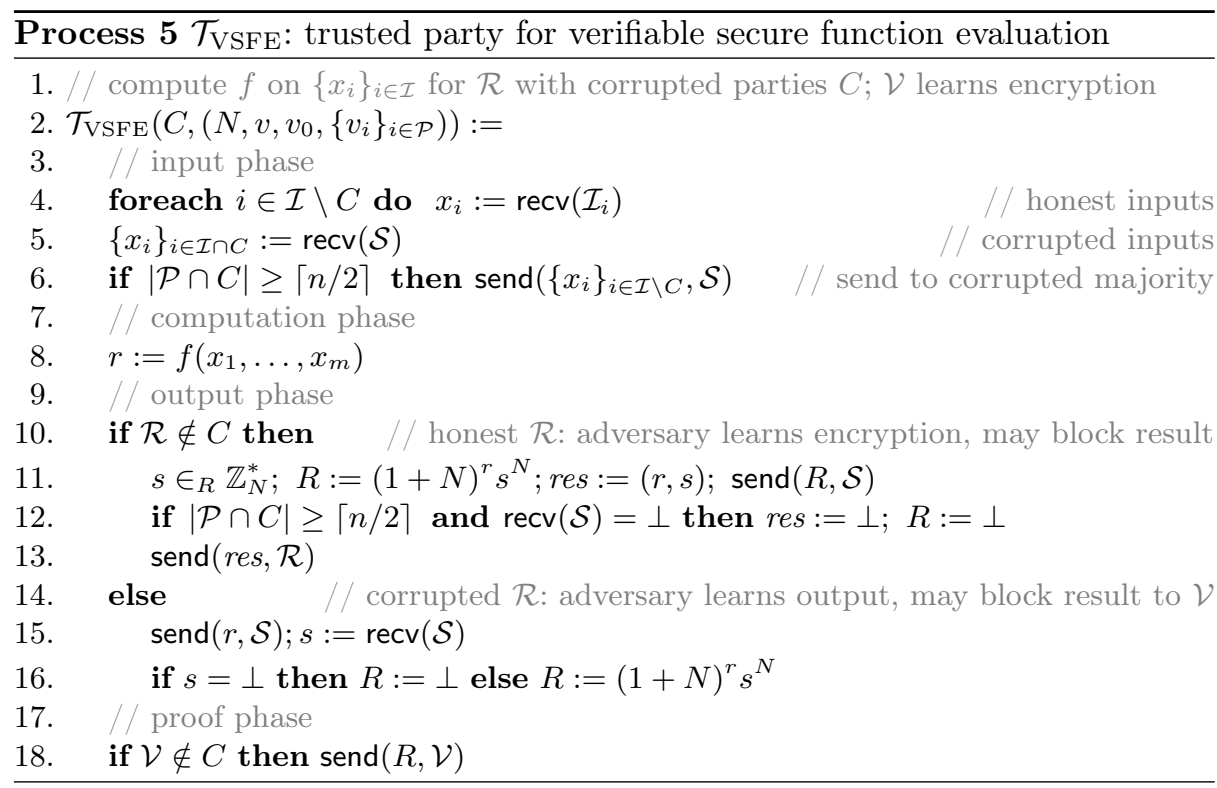

of all parties (including the adversary) and the set $\mathcal{O}$ of oracle queries and responses.

The ideal-world execution similarly involves $m$ input parties $i \in \mathcal{I}, n$ computation parties $i \in \mathcal{P}$, result party $\mathcal{R}$, verifier $\mathcal{V}$, and an adversary $\mathcal{S}$ corrupting parties $C \subset \mathcal{I} \cup \mathcal{P} \cup\{\mathcal{R}, \mathcal{V}\}$; but now, there is also an incorruptible trusted party $\mathcal{T}$. As before, the execution starts by setting up the keys (pk, $\left.\left\{s_{i}\right\}_{i \in \mathcal{P}}\right)$ of the Paillier cryptosystem. The input parties receive $x_{i}$ as input; the trusted party receives a list $C$ of corrupted parties and the public key pk. Then, it runs the code $\mathcal{T}_{\text {VSFE }}$ shown in Process 5, which we explain later. The adversary gets inputs (pk, $C,\left\{x_{i}\right\}_{i \in \mathcal{I} \cap C},\left\{s_{i}\right\}_{i \in \mathcal{P} \cap C}$ ), and outputs a value at will. In this model, there is no random oracle; instead, the adversary chooses the set $\mathcal{O}$ of oracle queries and responses (typically, those used to simulate a real-world adversary). As in the real-world case, $\operatorname{IDEAL}_{\mathcal{T}_{\mathrm{SFE}} \mathcal{S}}\left(k,\left(x_{1}, \ldots, x_{m}\right), C, a\right)$ is the random variable, given security parameter $k$, consisting of all parties' outputs and $\mathcal{O}$.

Definition 3. Protocol $\pi$ implements verifiable secure function evaluation in the random oracle model if, for every probabilistic polynomial time real-world adversary $\mathcal{A}$, there exists a probabilistic polynomial time ideal-world adversary $\mathcal{S}_{\mathcal{A}}$ such that, for all inputs $x_{1}, \ldots, x_{m}$; all sets of corrupted parties $C$; and all auxiliary

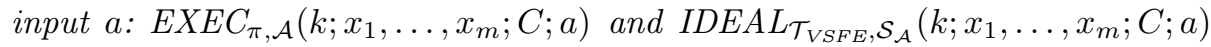
are computationally indistinguishable in security parameter $k$.

We remark that, while security in non-random-oracle secure function evaluation [Can98,CDN01] is preserved under (subroutine) composition, this is not the case for our random oracle variant. The reason is that our model and protocols assume that the random oracle is not used outside of the protocol. Using the random oracle model with dependent auxiliary input [Unr07,Wee09] might be 
enough to obtain a composition property; but adaptations are needed to make our protocol provably secure in that model. See Section 5 for a discussion.

We now discuss the trusted party $\mathcal{T}_{\text {VSFE }}$ for verifiable secure function evaluation. Whenever the computation succeeds, $\mathcal{T}_{\text {VSFE }}$ guarantees that the results are correct. Namely, $\mathcal{T}_{\mathrm{VSFE}}$ sends the result $r$ of the computation and randomness $s$ to $\mathcal{R}$ (line 13), and it sends encryption $(1+N)^{r} s^{N}$ of the result with randomness $s$ to $\mathcal{V}$ (line 18); if the computation failed, $\mathcal{R}$ gets $(\perp, \perp)$ and $\mathcal{V}$ gets $\perp .{ }^{4}$ Whether $\mathcal{T}_{\text {VSFE }}$ guarantees privacy (i.e., only $\mathcal{R}$ can learn the result) and robustness (i.e., the computation does not fail) depends on which parties are corrupted. Privacy and robustness with respect to $\mathcal{R}$ are guaranteed as long as only a minority of computation parties are corrupted. If not, then in line 6 , $\mathcal{T}_{\text {VSFE }}$ sends the honest parties' inputs to the adversary; and in line 12 , it gives the adversary the option to block the computation by sending $\perp$. Note that the adversary receives the inputs of the honest parties after it provides the inputs of the corrupted parties, so even if privacy is broken, the adversary cannot choose the corrupted parties' inputs based on the honest parties' inputs. For robustness with respect to $\mathcal{V}$, the result party needs to be honest. If not, then in line 15 , $\mathcal{T}_{\text {VSFE }}$ gives the adversary the option to block $\mathcal{V}$ 's result by sending $\perp$; in any case, it can choose the randomness. (Note that these thresholds are specific to CDN's "honest majority" setting; e.g., other protocols may satisfy privacy if all computation parties except one are corrupted.)

Note that this model does not cover the "universality" aspect of universally verifiable MPC. This is because the security model for secure function evaluation only covers the input/output behaviour of protocols, not the fact that "the verifier can be anybody". Hence, we design universally verifiable protocols by proving that they are verifiable, and then arguing based on the characteristics of the protocol (e.g., the verifier does not have any secret values) that this verifiability is "universal".

\subsection{Universally Verifiable CDN}

We now present the UVCDN protocol (Protocol 6) for universally verifiable secure function evaluation. At a high level, this protocol consists of the input, computation, and multiplication phases of the CDN protocol, with all proofs made non-interactive, followed by a new proof phase. As discussed, we can use the normal Fiat-Shamir (FS) heuristic in only a constant number of rounds; and we can use the multiparty FS heuristic only when it gives a "combined statement" that makes sense. Hence, we choose to use the FS heuristic for the proofs by the

\footnotetext{
4 Although we only guarantee computational indistinguishability and the verifier does not know what value is encrypted, this definition does guarantee that $\mathcal{V}$ receives the correct result. This is because the ideal-world output of the protocol execution contains $\mathcal{R}$ 's $r$ and $s$ and $\mathcal{V}$ 's $(1+N)^{r} s^{N}$, so a distinguisher between the ideal and real world can check correctness of $\mathcal{V}$ 's result. (If $s$ were not in $\mathcal{R}$ 's result, this would not be the case, and correctness of $\mathcal{V}$ 's result would not be guaranteed.) Also, note that although privacy depends on the security of the encryption scheme, correctness does not rely on any knowledge assumption.
} 


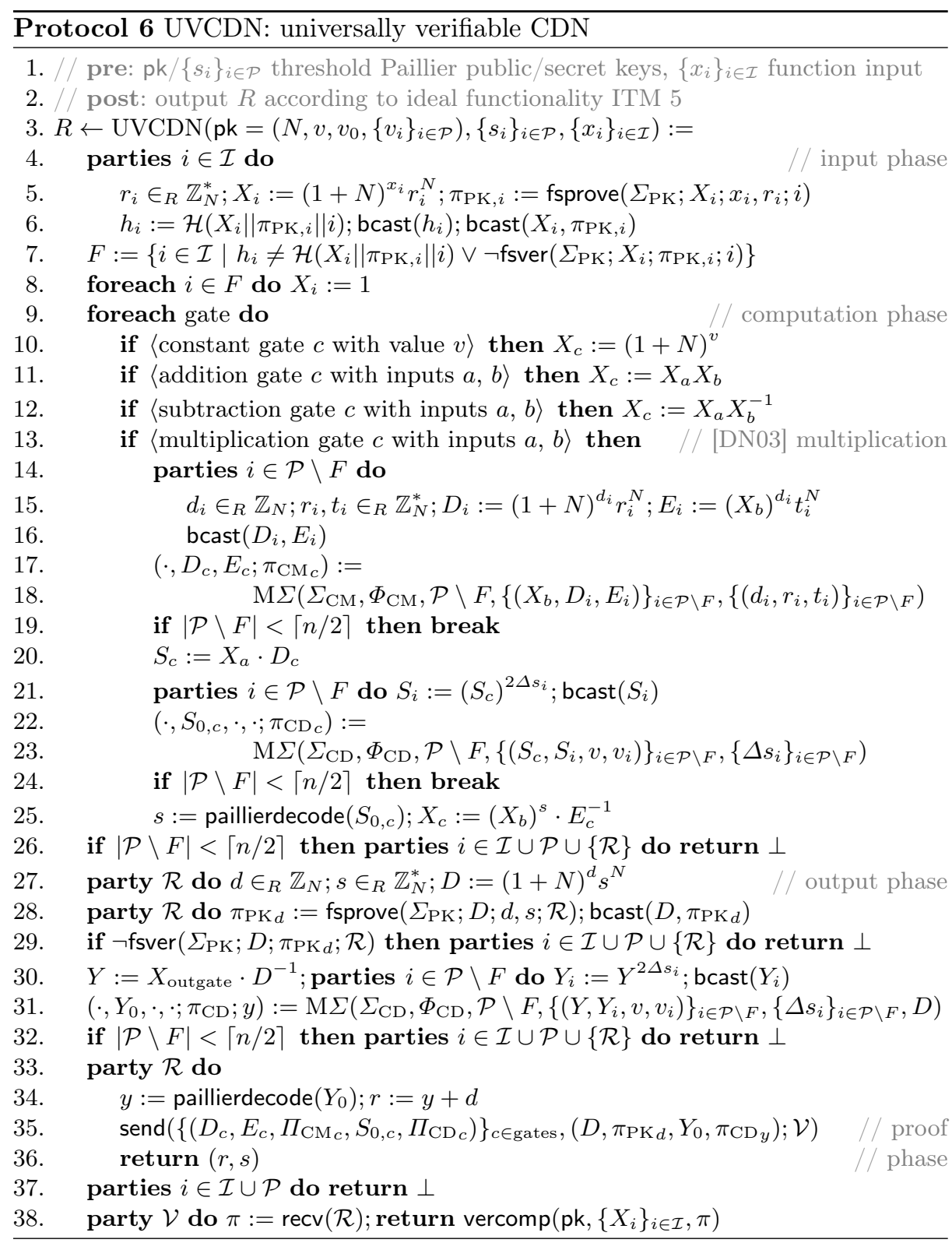

input and result parties, and the multiparty FS heuristic for the proofs by the computation parties.

In more detail, during the input phase of the protocol, the input parties provide their inputs (lines 4-8). As in the CDN protocol, each party encrypts its input and compiles a FS proof of knowledge (line 5). In the original CDN protocol, these encryptions and proofs would be broadcast directly; however, if a majority of computation parties are corrupted, then this allows corrupted parties 


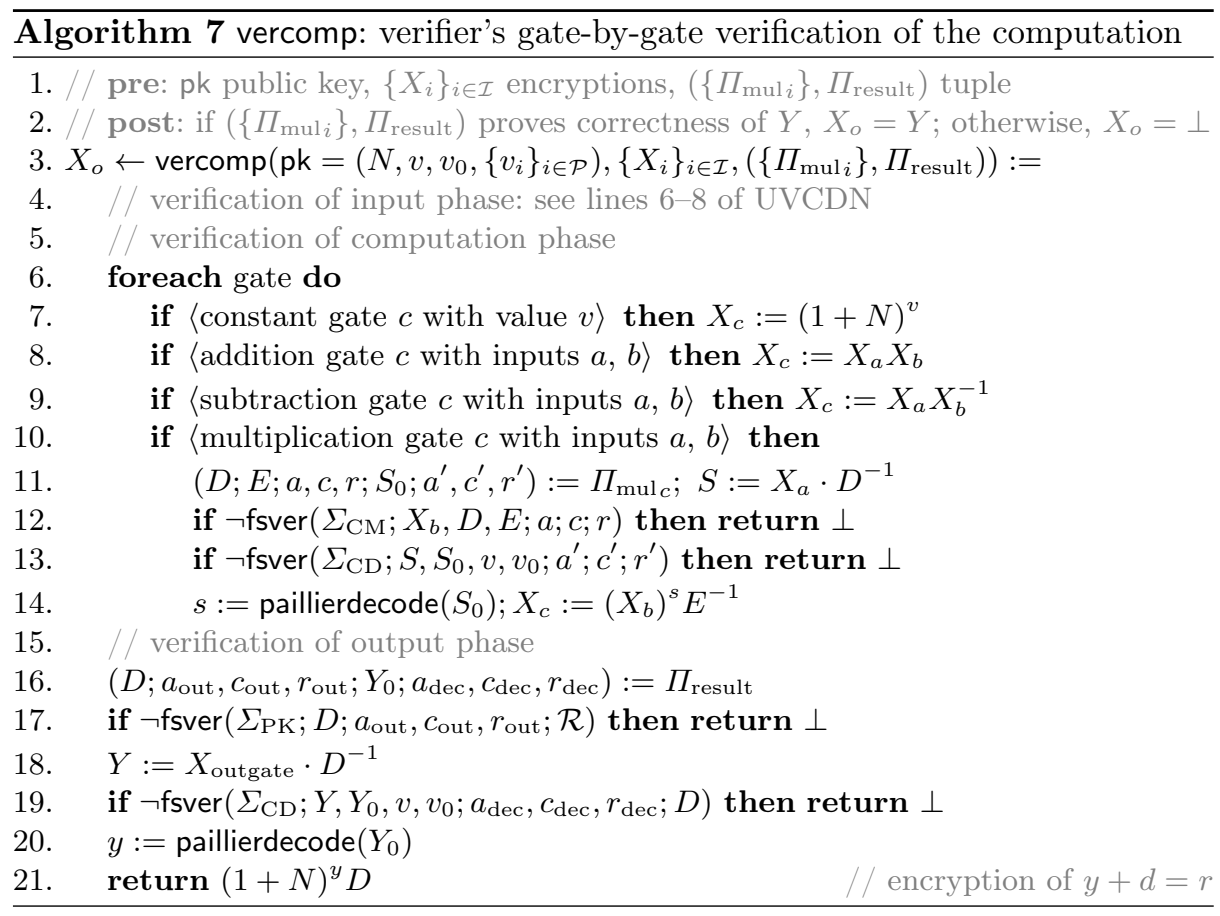

to adapt their inputs based on the inputs of the honest parties. To prevent this, we let each party first broadcast a hash of its input and proof; only after all parties have committed to their inputs using this hash are the actual encrypted inputs and proofs revealed (line 6). All parties that provide an incorrect hash or proof have their inputs set to zero (line 7-8).

The remainder of the computation follows the CDN protocol. During the computation phase, the function is evaluated gate-by-gate; for multiplication gates, the multiplication protocol from [DN03] is used, with proofs of correct multiplication and decryption using the multiparty FS heuristic (lines 14-25). During the output phase, the result party obtains the result by broadcasting an encryption of a random $d$ and proving knowledge using the normal FS heuristic (lines 27-28); the computation parties decrypt the result plus $d$, proving correctness using the multiparty FS heuristic (line 31). From this, the result party learns result $r$ (line 34); and it knows the intermediate values from the protocol and the proofs showing they are correct.

Finally, we include a proof phase in the UVCDN protocol in which the result party sends these intermediate values and proofs to the verifier (line 35). The verifier runs procedure vercomp (Algorithm 7) to verify the correctness of the computation (line 38). The inputs to this verification procedure are the public key of the Paillier cryptosystem; the encrypted inputs $\left\{X_{i}\right\}_{i \in \mathcal{I}}$ by the input parties; and the proof $\pi$ by the result party (which consists of proofs for each multiplication gate, and the two proofs from the output phase of the protocol). The verifier checks the proofs for each multiplication gate from the computation 
phase (lines 6-14); and the proofs from the output phase (lines 16-20), finally obtaining an encryption of the result (line 21). While not specified in vercomp, the verifier does also verify the proofs from the input phase: namely, in lines 7-8 of UVCDN, the verifier receives encrypted inputs and verifies their proofs to determine the encrypted inputs $\left\{X_{i}\right\}_{i \in \mathcal{I}}$ of the computation.

Apart from checking the inputs during the input phase, the verifier does not need to be present for the remainder of the computation until receiving $\pi$ from $\mathcal{R}$. This is what makes verification "universal": in practice, we envision that a trusted party publicly announces the Paillier public keys, and the input parties publicly announce their encrypted inputs with associated proofs: then, anybody can use the verification procedure to verify if a given proof $\pi$ is correct with respect to these inputs. In Appendix C, we prove that:

Theorem 1. Protocol UVCDN implements verifiable secure function evaluation in the random oracle model.

The proof uses two simulators: one for a honest majority of computation parties; one for a corrupted majority. The former simulator extends the one from [CDN01], obtaining privacy with a reduction to semantic security of the threshold Paillier cryptosystem. The latter does not guarantee privacy, and so can simulate the adversary by running the real protocol, ensuring correctness by witness-extended emulation.

\section{Concluding Remarks}

Our security model is specific to the CDN setting in two respects. First, we explicitly model that the verifier receives a Paillier encryption of the result (as opposed to another kind of encryption or commitment). We chose this formulation for concreteness; but our model generalises easily to other representations of the result. Second, it is specific to the setting where a minority of parties may be actively corrupted; but it is possible to change the model to other corruption models. For instance, it is possible to model the setting from [BDO14] where privacy is guaranteed when there is at least one honest computation party (and our protocols can be adapted to that setting). The combination of passively secure multiparty computation with universal verifiability is another interesting possible adaptation.

Our protocols are secure in the random oracle model "without dependent auxiliary input" [Wee09]. This means our security proofs assume that the random oracle has not been used before the protocol starts. Moreover, our simulator can only simulate logarithmically many sequential runs of our protocol due to technical limits of witness-extended emulation. These technical issues reflect the real-life problem that a verifier cannot see if a set of computation parties have just performed a computation, or they have simply replayed an earlier computation transcript. As discussed in [Unr07], both problems can be solved in practice by instantiating the random oracle with a keyed hash function, with every computation using a fresh random key. Note that all existing constructions require 
the random oracle model; achieving universally verifiable (or publicly auditable) multiparty computation in the standard model is open.

Several interesting variants of our protocol are possible. First, it is easy to achieve publicly auditable multiparty computation [BDO14] by performing a public decryption of the result rather than a private decryption for the result party. Another variant is basic outsourcing of computation, in which the result party does not need to be present at the time of the computation, but afterwards gets a transcript from which it can derive the computation result. Finally, it is possible to achieve universal verifiability using other threshold cryptosystems than Paillier. In particular, while the threshold ElGamal cryptosystem is much more efficient than threshold Paillier, it cannot be used directly with our protocols because it does not have a general decryption operation; but universally verifiable multiparty using ElGamal should still be possible by instead adapting the "conditional gate" variant of the CDN protocol from [ST04].

Finally, to close the loop, we note that our techniques can also be applied to reduce the cost of verification in universally verifiable voting schemes. Namely, for voting schemes relying on homomorphic tallying, we note that the $\Sigma$-proofs for correct decryption of the election result by the respective talliers can be combined into a single $\Sigma$-proof of constant size (independent of the number of talliers). Similarly, for voting schemes relying on mix-based tallying, the $\Sigma$ proofs for correct decryption of each vote by the respective talliers is reduced to a constant size per vote.

Acknowledgements The authors thank Sebastiaan de Hoogh, Thijs Laarhoven, and Niels de Vreede for useful discussions. This work was supported in part by the European Commission through the ICT program under contract INFSOICT-284833 (PUFFIN). The research leading to these results has received funding from the European Union Seventh Framework Programme (FP7/2007-2013) under grant agreement no 609611 (PRACTICE).

\section{References}

AABN08. M. Abdalla, J. H. An, M. Bellare, and C. Namprempre. From Identification to Signatures Via the Fiat-Shamir Transform: Necessary and Sufficient Conditions for Security and Forward-Security. IEEE Transactions on Information Theory, 54(8):3631-3646, 2008.

$\mathrm{ACG}^{+}$14. P. Ananth, N. Chandran, V. Goyal, B. Kanukurthi, and R. Ostrovsky. Achieving Privacy in Verifiable Computation with Multiple Servers - Without FHE and without Pre-processing. In Proceedings of PKC '14, volume 8383 of Lecture Notes in Computer Science, pages 149-166. Springer, 2014.

$\mathrm{BCD}^{+}$09. P. Bogetoft, D. L. Christensen, I. Damgård, M. Geisler, T. P. Jakobsen, M. Krøigaard, J. D. Nielsen, J. B. Nielsen, K. Nielsen, J. Pagter, M. I. Schwartzbach, and T. Toft. Secure Multiparty Computation Goes Live. In Proceedings of FC'09, volume 5628 of Lecture Notes in Computer Science, pages 325-343. Springer, 2009.

BDO14. C. Baum, I. Damgård, and C. Orlandi. Publicly Auditable Secure MultiParty Computation. In Proceedings of SCN '14, volume 8642 of Lecture Notes in Computer Science, pages 175-196. Springer, 2014. 
BR93. M. Bellare and P. Rogaway. Random Oracles are Practical: A Paradigm for Designing Efficient Protocols. In Proceedings of CCS '93, pages 62-73. ACM, 1993.

Can98. R. Canetti. Security and Composition of Multi-party Cryptographic Protocols. Journal of Cryptology, 13:2000, 1998.

CDN01. R. Cramer, I. Damgård, and J. Nielsen. Multiparty Computation from Threshold Homomorphic Encryption. In Proceedings of EUROCRYPT '01, volume 2045 of Lecture Notes in Computer Science, pages 280-300. Springer, 2001.

CF85. J. Cohen and M. Fischer. A Robust and Verifiable Cryptographically Secure Election Scheme. In Proceedings of FOCS '85, pages 372-382. IEEE, 1985.

Des93. Y. Desmedt. Threshold cryptosystems. In Proceedings of AUSCRYPT '92, volume 718 of Lecture Notes in Computer Science, pages 1-14. Springer, 1993.

dH12. S. de Hoogh. Design of large scale applications of secure multiparty computation: secure linear programming. $\mathrm{PhD}$ thesis, Eindhoven University of Technology, 2012.

DJ01. I. Damgård and M. Jurik. A Generalisation, a Simplification and Some Applications of Paillier's Probabilistic Public-Key System. In Proceedings of PKC '01, volume 1992 of Lecture Notes in Computer Science, pages 119-136. Springer, 2001.

DN03. I. Damgård and J. B. Nielsen. Universally Composable Efficient Multiparty Computation from Threshold Homomorphic Encryption. In Proceedings of CRYPTO '03, volume 2729 of Lecture Notes in Computer Science, pages 247-264. Springer, 2003.

DPSZ12. I. Damgård, V. Pastro, N. Smart, and S. Zakarias. Multiparty Computation from Somewhat Homomorphic Encryption. In Proceedings of CRYPTO '12, volume 7417 of Lecture Notes in Computer Science, pages 643-662. Springer, 2012.

EFLL12. Y. Ejgenberg, M. Farbstein, M. Levy, and Y. Lindell. SCAPI: The Secure Computation Application Programming Interface. IACR Cryptology ePrint Archive, 2012:629, 2012.

FGP14. D. Fiore, R. Gennaro, and V. Pastro. Efficiently Verifiable Computation on Encrypted Data. In Proceedings of CCS '14, pages 844-855. ACM, 2014.

GK03. S. Goldwasser and Y. T. Kalai. On the (In)security of the Fiat-Shamir Paradigm. In Proceedings of FOCS '03, pages 102-113. IEEE Computer Society, 2003.

GKP $^{+}$13. S. Goldwasser, Y. T. Kalai, R. A. Popa, V. Vaikuntanathan, and N. Zeldovich. Reusable garbled circuits and succinct functional encryption. In Proceedings of STOC '13, pages 555-564. ACM, 2013.

Gro04. J. Groth. Evaluating Security of Voting Schemes in the Universal Composability Framework. In Proceedings of ACNS '04, volume 3089 of Lecture Notes in Computer Science, pages 46-60. Springer, 2004.

IPS09. Y. Ishai, M. Prabhakaran, and A. Sahai. Secure Arithmetic Computation with No Honest Majority. In Proceedings of TCC '09, volume 5444 of Lecture Notes in Computer Science, pages 294-314. Springer, 2009.

Jur03. M. J. Jurik. Extensions to the Paillier Cryptosystem with Applications to Cryptological Protocols. PhD thesis, University of Aarhus, 2003.

KMR12. M. Keller, G. L. Mikkelsen, and A. Rupp. Efficient Threshold ZeroKnowledge with Applications to User-Centric Protocols. In Proceedings of ICITS 2012, volume 7412 of Lecture Notes in Computer Science, pages 147-166. Springer, 2012. 
NKDM03. A. Nicolosi, M. N. Krohn, Y. Dodis, and D. Mazières. Proactive TwoParty Signatures for User Authentication. In Proceedings of NDSS 2003. The Internet Society, 2003.

Pai99. P. Paillier. Public-Key Cryptosystems Based on Composite Degree Residuosity Classes. In Proceedings of EUROCRYPT '99, volume 1592 of Lecture Notes in Computer Science, pages 223-238. Springer, 1999.

PHGR13. B. Parno, J. Howell, C. Gentry, and M. Raykova. Pinocchio: Nearly Practical Verifiable Computation. In Proceedings of SEP 2013, pages 238-252. IEEE, 2013.

Sch89. C. Schnorr. Efficient Identification and Signatures for Smart Cards. In Proceedings of CRYPTO '89, volume 435 of Lecture Notes in Computer Science, pages 239-252. Springer, 1989.

SK95. K. Sako and J. Kilian. Receipt-Free Mix-Type Voting Scheme-A Practical Solution to the Implementation of a Voting Booth. In Proceedings of EUROCRYPT '95, volume 921 of Lecture Notes in Computer Science, pages 393-403. Springer, 1995.

ST04. B. Schoenmakers and P. Tuyls. Practical Two-Party Computation Based on the Conditional Gate. In Proceedings of ASIACRYPT '04, volume 3329 of Lecture Notes in Computer Science, pages 119-136. Springer, 2004.

ST06. B. Schoenmakers and P. Tuyls. Efficient Binary Conversion for Paillier Encrypted Values. In Proceedings of EUROCRYPT '06, volume 4004 of Lecture Notes in Computer Science, pages 522-537. Springer, 2006.

Unr07. D. Unruh. Random Oracles and Auxiliary Input. In Proceedings of CRYPTO '0\%, volume 4622 of Lecture Notes in Computer Science, pages 205-223. Springer, 2007.

WB13. M. Walfish and A. J. Blumberg. Verifying computations without reexecuting them: from theoretical possibility to near-practicality. Electronic Colloquium on Computational Complexity, 20:165, 2013.

Wee09. H. Wee. Zero Knowledge in the Random Oracle Model, Revisited. In Proceedings of ASIACRYPT '09, volume 5912 of Lecture Notes in Computer Science, pages 417-434. Springer, 2009.

\section{A Simulation-Based Security in the Random Oracle Model}

We prove our protocols secure using the simulation paradigm in the random oracle model [BR93,Wee09]. In the random oracle model, evaluations of hash function $\mathcal{H}:\{0,1\}^{*} \rightarrow\{0,1\}^{2 l}$ are modelled as queries to a "random oracle" $\mathcal{O}$ that evaluates a perfectly random function. When simulating an adversary that operates in the random oracle model, the simulator also simulates the random oracle with respect to the adversary. In particular, it can choose how to respond to the adversary's queries (but, to achieve security, it should provide random values so that the adversary cannot distinguish between the real world and the simulation based on the output of the random oracle).

More precisely, we work in the explicitly programmable random oracle model without dependent auxiliary input [Wee09]. The random oracle is seen as a partial function that initially has an empty codomain (i.e., it is "without dependent auxiliary input"). In a real-world execution in this model, both the honest parties and the adversary use the random oracle for hash function evaluations. Namely, 


$$
\begin{array}{ll}
\operatorname{dom}(\mathcal{O}), \operatorname{codom}(\mathcal{O}), \operatorname{rng}(\mathcal{O}) & \text { Domain/codomain/range of random oracle } \mathcal{O}, \\
& \text { seen as partial function } \\
\text { fail } & \text { Terminate simulation, returning special error value } \\
\mathcal{A}, \mathcal{O} & \text { Global variable: simulated attacker/random oracle } \\
v:=\mathcal{A}^{\mathcal{O}}(w) & \text { Exchange values } v, w \text { with } \mathcal{A} \text { having oracle access to } \mathcal{O}
\end{array}
$$

Fig. 2. Notation and conventions for simulation-based security in the Random Oracle Model

when a party calls the oracle on a value $v \in \operatorname{dom}(\mathcal{O})$, it receives $\mathcal{O}(v)$; otherwise, a fresh random value is generated and $\mathcal{O}$ is updated accordingly. At the end of the execution, $\mathcal{O}$ contains all pairs of oracle queries made during the execution and their responses. In an ideal-world execution, the simulator can directly modify the pre-image/image pairs in $\mathcal{O}$; the simulated adversary only has oracle access to $\mathcal{O}$ as in the real-world execution. Again, at the end of the simulation, $\mathcal{O}$ contains all values on which the oracle has been set. Computational (or statistical) indistinguishability between real and simulated executions is defined [Wee09] by stating that no p.p.t. (or unbounded) algorithm can distinguish them, where the distinguisher has oracle access to $\mathcal{O}$. We prove slightly stronger versions of indistinguishability; namely, instead of giving the distinguisher oracle access to $\mathcal{O}$, we simply supply it with the full list $\mathcal{O}$. We can then simply use the normal, non-oracle, definitions for indistinguishability; this is clearly at least as strong.

Our proofs rely on the absence of dependent auxiliary input. When a party presents a non-interactive proof of knowledge, we perform rewinding to find the witness to that proof; but if oracle queries before the protocol execution are allowed, then a party may replay a proof that was performed before the protocol execution, making rewinding impossible. As noted in [Unr07], this suggests that the random oracle in our protocols should be instantiated with a keyed hash function, where every protocol instance uses a different key. See Section 5 for a discussion.

Figure 2 lists the notation we use when presenting simulators in the random oracle model. We use global variables $\mathcal{A}$ and $\mathcal{O}$ to denote the current state of the adversary and the random oracle. An invocation of $\mathcal{A}$ with oracle access to $\mathcal{O}$ is denoted $v:=\mathcal{A}^{\mathcal{O}}(w)$; afterwards, both $\mathcal{O}$ and $\mathcal{A}$ are updated to reflect the respective new states.

\section{A.1 The Fiat-Shamir Heuristic and Witness-Extended Emulation}

When the Fiat-Shamir heuristic (Section 3.1) is used, in some situations a simulator can extract witnesses from proofs by corrupted parties. Namely, in [Gro04], Groth showed that, by simulating an adversary using witness-extended emulation, a simulator can obtain witnesses for all proofs that the adversary produces. Specifically, Groth proved the following:

Theorem 2 ([Gro04]). Let $\left(\mathcal{A}^{\prime}, \mathcal{O}^{\prime}, \boldsymbol{x}, \boldsymbol{p}\right) \leftarrow \mathcal{A}^{\mathcal{O}}(z)$ be an adversary $\mathcal{A}$ interacting with random oracle $\mathcal{O}$ that, on some polynomial-length input $z$, outputs a list $\boldsymbol{x}$ of statements, and a list $\boldsymbol{p}$ of corresponding validating Fiat-Shamir proofs. 
Then there exists a p.p.t. emulator $\left(\mathcal{A}^{\prime}, \mathcal{O}^{\prime}, \boldsymbol{x}, \boldsymbol{p}, \boldsymbol{w}\right) \leftarrow \mathcal{E}_{\mathcal{A}}^{\mathcal{O}}(z)$ such that the part $\left(\mathcal{A}^{\prime}, \mathcal{O}^{\prime}, \boldsymbol{x}, \boldsymbol{p}\right)$ of the output of $\mathcal{E}_{\mathcal{A}}^{\mathcal{O}}$ is perfectly indistinguishable from the output of $\mathcal{A}$, and $\boldsymbol{w}$ are witnesses corresponding to the statements $\boldsymbol{x}$.

Essentially, the witness-extended emulator $\mathcal{E}_{\mathcal{A}}^{\mathcal{O}}$ simulates the adversary $\mathcal{A}$, keeping track of all oracle queries it makes. For each valid proof that $\mathcal{A}$ produces, it rewinds $\mathcal{A}$ to the point of the oracle query used to obtain the challenge, and keeps on reprogramming the random oracle until $\mathcal{A}$ again produces a correct proof with a new challenge. It finally extracts the witness using the special soundness property of the $\Sigma$-protocol.

However, a major limitation of the technique of [Gro04] is that it only considers a single invocation of the adversary. On the other hand, the CDN protocol consists of different rounds in which the adversary is invoked with inputs from the honest parties. Now, if the adversary provides a proof in its $r$ th invocation, it may have already queried the random oracle for the announcement of that proof in an earlier invocation $s<r$. Then, to extract the witness for the proof from invocation $r$, we need to simulate the adversary from invocation $s$. This means that we also need to re-compute the messages from the honest party to the adversary for all rounds between $s$ and $r$. However, computing these messages in general requires witnesses for the proofs of the adversary between rounds $s$ and $r$. Hence, to extract the witnesses for the $r$ th invocation, we need to recursively extract witnesses for all rounds between $s$ and $r$, which in turn may also require recursive rewinding. Hence, if Fiat-Shamir proofs take place in $R$ invocations of the adversary, then witness-extended emulation may increase the running time of a simulator by a factor $O(R !)$. Because we need simulators to be polynomial time , $R$ ! should be polynomial in the security parameter, so we can use the Fiat-Shamir heuristic, but only in essentially a constant number of rounds (as in our UVCDN protocol, where we only use the normal Fiat-Shamir heuristic at the beginning and the end of the protocol).

Our multiparty Fiat-Shamir heuristic (Section 3.2) addresses the above limitations while also combining proofs of individual parties into one single proof for a combined statement. If this combination is not desired, then each party can simply hash the concatenation of all parties' announcements instead of taking combinations $\Phi$.stmt $\left(\left\{v_{i}\right\}_{i \in P \backslash F}\right), \Phi$.ann $\left(\left\{a_{i}\right\}_{i \in P \backslash F}\right.$. Note that also in this case, the extra round in which parties commit to their announcements is still needed. Without this round, the adversary could choose its announcement after the honest parties chose theirs, and hence, the simulator would not know on which pre-image to program the random oracle when simulating the honest parties' proofs. In the non-combination case, the parties could alternatively broadcast their announcements; and then use a hash of the concatenation of the announcements and some fresh randomness. This way, the simulator can simulate the honest parties' proofs because the adversary cannot predict the pre-image before seeing the proof; while the adversary cannot make oracle queries for its own proofs too early because it needs to wait for the honest parties' announcements. Another way of addressing the limitations in the non-combination case would be to add the full previous communication transcript to the hash (or, in any 
case, some recent messages that contain sufficient entropy by honest parties): this way, rewinding is guaranteed to be to a recent moment in the protocol.

\section{B Simulator of the Multiparty Fiat-Shamir Heuristic}

Our simulator $\mathcal{S}_{\mathrm{M} \Sigma}$ of the multiparty Fiat-Shamir heuristic is shown in Algorithm 8. We now explain the general idea, deferring the discussion of exceptional cases to the security proof.

As long as flag stage2 is not set, the simulator behaves like the protocol. First, the parties exchange hashed commitments (line 10-12). Namely, the simulator generates random hash values for the honest parties (line 10), and receives hash values for the corrupted parties (line 11). Note that the adversary can later open these values only if they come from the random oracle, hence the simulator knows the adversary's pre-images $a_{i}$ (line 12). Next, the parties open their commitments (lines 16-26). The simulator generates a random challenge $c$ (line 16); simulates the $\Sigma$-protocol for the honest parties (line 18); and programs the random oracle so that the announcements of the honest parties hash to the values $h_{i}$ supplied earlier (lines 19-20). If possible, the simulator combines the announcements $a_{i}$ of the honest and corrupted parties, and programs the random oracle to return $c$ on the result (lines 21-24). The simulator then the receives announcements $a_{i}^{\prime}$ for the corrupted parties (line 25); and checks if they are correct (line 26); if so, it exchanges responses to the challenge (line 29). If all parties provided correct responses, then the protocol terminates: in this case, the simulator stores the state at this point for returning it when the simulation ends, and sets flag stage2 (line 33). Otherwise, the simulator continues repeats the above process with the parties that have not misbehaved so far (line 40).

When flag stage2 is set, the simulator has simulated one successful run of the multiparty Fiat-Shamir heuristic for which it will now extract witnesses. Note that variables $\mathcal{A}_{\text {start }}, \mathcal{O}_{\text {start }}$, and $F^{\prime}$ contain the state of the protocol at the point when the adversary has supplied hash values $h_{i}$ for which the simulator knows the pre-images $a_{i}$, and a challenge $c^{\prime}$ with correct responses $\left\{r_{i}^{\prime}\right\}_{i \in\left(P \backslash F^{\prime}\right) \cap C}$. To extract witnesses for the parties in $\left(P \backslash F^{\prime}\right) \cap C$, the simulator keeps re-winding to state $\left(\mathcal{A}_{\text {start }}, \mathcal{O}_{\text {start }}, F^{\prime}\right)$ (line 15$)$, and repeats the above simulation procedure (lines 16-30), until the adversary has again produced correct responses for the same parties (line 31). In this case, it has responses from the adversary for the same announcement with different challenges, from which it can extract witnesses using the special soundness property of the $\Sigma$-protocol (line 37 ). Finally, the simulator returns the adversary state after the first successful protocol run, along with the proof and the witnesses for the contributing corrupted parties (line 39).

We now prove that simulator $\mathcal{S}_{\mathrm{M} \Sigma}$ satisfies soundness and zero-knowledgeness. The statement of our lemma is analogous to the statement from [CDN01], with two technical differences. First, we do not use trapdoor commitments, in effect replacing them by the random oracle. Second, we do not guarantee perfect indistinguishability because our simulator may occasionally fail. We get the following: 


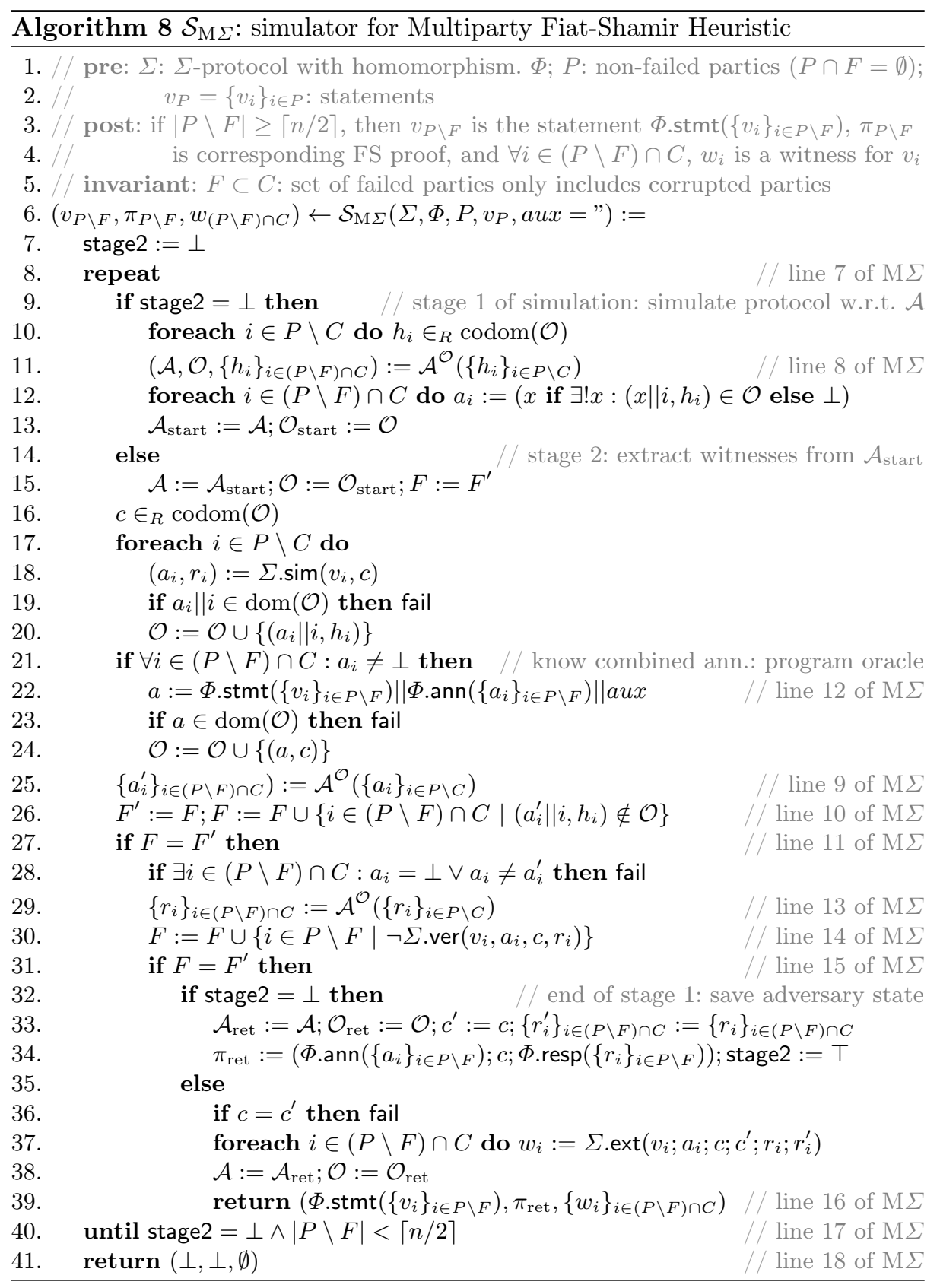

Lemma 1. Assume that fewer than $\lceil n / 2\rceil$ parties are corrupted. Define realworld executions of $M \Sigma$ and ideal-world executions of $\mathcal{S}_{M \Sigma}$ as follows:

- Let $\left(\mathcal{A}^{\prime}, \mathcal{O}^{\prime}, F^{\prime}, v_{P \backslash F^{\prime}}, \pi_{P \backslash F^{\prime}}\right) \leftarrow \operatorname{exec}_{M \Sigma}\left(\mathcal{A}, \mathcal{O}, F, \Sigma, \Phi, P, v_{P}, w_{P}\right.$, aux $) \operatorname{de}$ note a run of the $M \Sigma$ protocol (Protocol 4) with initial adversary state $\mathcal{A}$, 
random oracle $\mathcal{O}$, set $F$ of failed parties, parameters $\left(\Sigma, \Phi, P, v_{P}, w_{P}\right.$, aux $)$, final states $\mathcal{A}^{\prime}, \mathcal{O}^{\prime}, F$, and return values $v_{P \backslash F}, \pi_{P \backslash F}$.

- Let $\left(\mathcal{A}^{\prime}, \mathcal{O}^{\prime}, F^{\prime}, v_{P \backslash F^{\prime}}, \pi_{P \backslash F^{\prime}}, w_{(P \backslash F) \cap C}\right) \leftarrow \operatorname{sim}_{\mathcal{S}_{M \Sigma}}\left(\mathcal{A}, \mathcal{O}, F, \Sigma, \Phi, P, v_{P}\right.$, aux $)$ denote a run of the $\mathcal{S}_{M \Sigma}$ simulator with initial adversary state $\mathcal{A}$, random oracle state $\mathcal{O}$, and set $F$ of failed parties, and parameters $\left(\Sigma, \Phi, P, v_{P}\right.$, aux $)$; and final states $\mathcal{A}^{\prime}, \mathcal{O}^{\prime}, F^{\prime}$ and return values $v_{P \backslash F^{\prime}}, \pi_{P \backslash F^{\prime}}, w_{\left(P \backslash F^{\prime}\right) \cap C}$.

Then $\mathcal{S}_{M \Sigma}$ is a p.p.t. algorithm satisfying the following two properties:

Soundness Except with negligible probability, $w_{\left(P \backslash F^{\prime}\right) \cap C}=\left\{w_{i}\right\}_{i \in\left(P \backslash F^{\prime}\right) \cap C}$ are valid witnesses for the statements $v_{i}$ of the corrupted parties in $P$ that produced verifying proofs.

Zero-Knowledgeness The part $\left(\mathcal{A}^{\prime}, \mathcal{O}^{\prime}, F^{\prime}, v_{P \backslash F^{\prime}}, \pi_{P \backslash F^{\prime}}\right)$ of the output of $\operatorname{sim}_{\mathcal{S}_{M \Sigma}}$ is statistically indistinguishable from the output of exec $\mathrm{M \Sigma}$.

Proof. We need to show that $\mathcal{S}_{\mathrm{M} \Sigma}$ runs in polynomial time, and that it satisfies soundness and zero-knowledgeness.

First, we show that the simulator only fails with negligible probability: It fails in line 19 if the random oracle has already been programmed on a simulated announcement. This happens with negligible probability because of the nontriviality property of the $\Sigma$-protocol. It fails in line 23 if the random oracle has already been programmed on a homomorphically combined announcement. This happens with negligible probability because of the randomness property of the homomorphism $\Phi$. It fails in line 28 if the adversary manages to supply a preimage $a_{i}^{\prime}$ different from the one calculated in line 12 . Then the adversary has found a collision or it has found a pre-image of $h_{i}$ without getting $h_{i}$ from the oracle, which happens with negligible probability. Finally, it fails in line 36 if the simulator has twice generated the same challenge randomly in the codomain of the random oracle. But this codomain is $\{0,1\}^{2 l}$ with $l$ a security parameter, so also this happens with negligible probability.

For soundness: the adversary returns values $w_{i}=\Sigma \operatorname{ext}\left(v_{i}, a_{i}, c, c^{\prime}, r_{i}, r_{i}^{\prime}\right)$ for which we know that $\Sigma \cdot \operatorname{ver}\left(v_{i}, a_{i}, c, r_{i}\right)$ and $\Sigma \cdot \operatorname{ver}\left(v_{i}, a_{i}, c^{\prime}, r_{i}^{\prime}\right)$ hold (by line 30), and $c \neq c^{\prime}$ (by line 36). Hence, the $w_{i}$ are valid witnesses by the special soundness property of the $\Sigma$-protocol.

For zero-knowledgeness, note that the part $\left(\mathcal{A}^{\prime}, \mathcal{O}^{\prime}, F^{\prime}, v_{P \backslash F^{\prime}}, \pi_{P \backslash F^{\prime}}\right)$ returned by the simulated execution is determined while stage $2=\perp$. Namely, one checks that $\mathcal{A}^{\prime}$ and $\mathcal{O}^{\prime}$ are $\mathcal{A}_{\text {ret }}$ and $\mathcal{O}_{\text {ret }}$ as set in line $33 ; F^{\prime}$ is the value $F$ when line 33 was executed; $v_{P \backslash F^{\prime}}$ depends only on that value $F^{\prime}$; and $\pi_{P \backslash F^{\prime}}$ is set in line 34. But observe that, while stage $2=\perp$, the simulator behaves like the original protocol with respect to the adversary, except in three ways. The first is that the protocol may fail, but as shown, this happens only with negligible probability so it does not affect statistical indistinguishability. The second is that, in line 26 of the simulator, we check if $\left(a_{i}^{\prime} \| i, h_{i}\right) \in \mathcal{O}$ instead of querying the random oracle. In the protocol, it might be that $a_{i}^{\prime} \| i$ was not queried before, but when queried, the random oracle happens to return $h_{i}$. Again, this happens with only negligible probability. Finally, all proofs of the honest parties are simulated. The special honest-verifier zero-knowledgeness property of the $\Sigma$-protocol now 
implies that $\left(\mathcal{A}^{\prime}, \mathcal{O}^{\prime}, F^{\prime}, v_{P \backslash F^{\prime}}, \pi_{P \backslash F^{\prime}}\right)$ is identically distributed in the real and simulated protocol run.

Finally, we need to show that the simulator runs in polynomial time. For this, let us analyse how often the main repeat... until loop (lines 8-40) is executed. If stage2 $=\perp$ at the beginning of the loop, then either $|P \backslash F|$ becomes strictly smaller, or stage 2 is set to $T$. Hence, the time spent while stage $2=\perp$ is certainly polynomial. Now, consider the loop execution in which stage2 is set to $T$, i.e., in which all provers provide correct announcements and responses for the first time. Let $\epsilon$ be the a priori probability, from line 16 of $\mathcal{S}_{\mathrm{M} \Sigma}$, that all provers indeed provide correct announcements and responses. Then with probability $\epsilon$, line 32 is reached; and afterwards, the loop is executed from the same state (line 15) until we again get correct announcements and proofs, which takes an expected $1 / \epsilon$ number of tries. Hence, the loop is executed with stage $2=\top$ with probability $\epsilon$ for an expected $1 / \epsilon$ number of times, hence it contributes polynomially to the running time. This completes the proof.

Note that the above proof relies on the fact that communication happens on a broadcast channel. Namely, in this case, all honest parties simulate their $\Sigma$-protocol based on the same challenge. However, we remark that the simulator could also be made to work in a non-broadcast setting. In this case, in line 12 , it determines announcements of the corrupted parties with respect to each honest party; and based on that, decides for how many different challenges to simulate the honest parties' $\Sigma$-protocols.

\section{Security of the UVCDN Protocol: Proof of Theorem 1}

To prove security of the UVCDN protocol, we need to build a simulator for every adversary $\mathcal{A}$. Because the honest majority and corrupted majority cases are very different, we define two simulators: simulator $\mathcal{S}_{\text {UVCDN }}^{\text {honest }}$ (Process 9 ) for adversaries that corrupt a minority of computation parties; and simulator $\mathcal{S}_{\text {UVCDN }}^{\text {corrupt }}$ (Process 10) for adversaries that corrupt a majority. Below, we present the simulators and prove that they correctly simulate the protocol in their respective cases. Theorem 1 follows directly from Lemmas 2 and 3 below.

\section{C.1 The Honest Majority Case}

If fewer than $\lceil n / 2\rceil$ computation parties are corrupted, then we guarantee privacy like the original CDN protocol. Our simulator $\mathcal{S}_{\text {UVCDN }}^{\text {honest }}$ (Process 9) for this case is an adaptation of CDN simulator from [CDN01]. This simulator follows the CDN simulator except for simulating non-interactive proofs rather than interactive proofs; UVCDN's modified input phase; the improved [DN03] multiplication protocol rather than the original protocol from [CDN01]; and UVCDN's proof phase. Non-interactive proofs using the multiparty Fiat-Shamir heuristic are simulated by simulator $\mathcal{S}_{\mathrm{M} \Sigma}$ (Algorithm 8) whose correctness we have proven in Appendix B.

Compared to CDN's input phase, the input phase of UVCDN has an extra round (line 6 of the protocol) in which the input parties first commit to their 


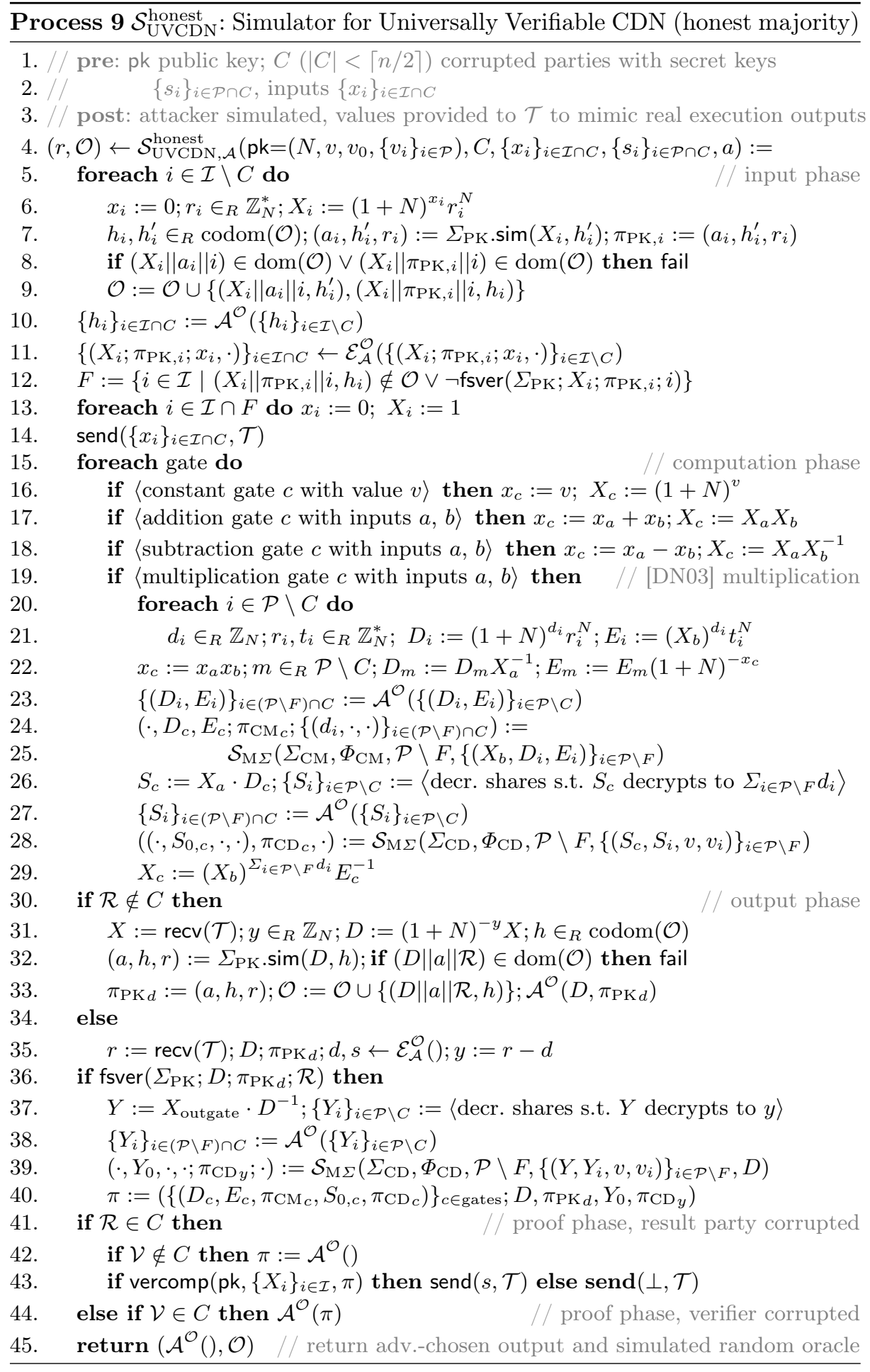


encrypted inputs and proofs of knowledge. For the simulator $\mathcal{S}_{\text {UVCDN }}^{\text {honest }}$, this extra round makes little difference: as in the CDN simulator, it produces the encryptions, proofs, and commitments of the honest parties using zero for their inputs (line 6-9). ${ }^{5}$ Because of the non-interactive proofs, we use witness-extended emulation to extract the inputs of the corrupted party. Namely, in line $10, \mathcal{S}_{\text {UVCDN }}^{\text {honest }}$ first obtains the corrupted commitments from $\mathcal{A}$. The next invocation of the adversary would return the encryptions and proofs of knowledge; by calling the witness-extended emulator $\mathcal{E}_{\mathcal{A}}^{\mathcal{O}}$, we additionally get the witnesses for these proofs, i.e., the plaintexts of the encryptions (line 11). (As discussed, this call includes possible recursive calls to the simulator.) These can then be provided to the trusted party (line 14).

Lines 20-29 of $\mathcal{S}_{\text {UVCDN }}^{\text {honest }}$ show our simulation of the improved multiplication protocol from [DN03]. Analogously to Theorem 3 of [CDN01], lines 20-29 simulate multiplication given encryptions $X_{a}, X_{b}$ of the values to be multiplied, and plaintext $x_{c}$ of the result ${ }^{6}$. This simulation is constructed to be statistically indistinguishable from the multiplication protocol itself (lines 14-25 of UVCDN). The simulator generate values $d_{i}, r_{i}, t_{i}, D_{i}, E_{i}$ on behalf of the honest parties as in the protocol (lines 20-21); but then multiplies one of the resulting $\left(D_{i}, E_{i}\right)$ by $X_{a}^{-1}$ and $(1+N)^{-x_{c}}$, respectively (line 22). This way, the simulator knows that $S_{c}=X_{a} \cdot D_{c}=X_{a} \cdot \prod_{i \in \mathcal{P} \backslash F} D_{i}$ computed in line 26 encrypts $\sum_{i \in \mathcal{P} \backslash F} d_{i}$. It simulates the proofs of correct multiplication of the $\left(D_{i}, E_{i}\right)$ (line 24-25); and computes $S_{c}$ as in the protocol (line 26). Finally, it simulates decryption of $S_{c}$ to $\sum_{i \in \mathcal{P} \backslash F} d_{i}$ (line 28), and computes $X_{c}$ as in the protocol (line 29). One checks that this is indeed a statistically indistinguishable simulation.

Our simulation of the output and proof phases (line 30-44 of the simulator) differs from the simulation in [CDN01]. Namely, in our case, simulation of the output phase results in an encryption of the actual result of the computation (obtained from the trusted party), whereas the CDN simulator works with a simulated encryption until the end. Indeed, if the result party is honest, our simulator receives an encryption $X$ of the actual result (line 31 ). Recall that the verifier sets the encrypted computation result to $(1+N)^{y} D$ with $D$ provided by the result party, so we simulate $D$ from the result party as $D=(1+N)^{-y} X$ to ensure that the verifier's output will be $X$. If the result party is corrupted, the simulator receives $D$ from the adversary. To ensure that $(1+N)^{y} D$ indeed encrypts the result of the computation, the simulator extracts plaintext $d$ and randomness $s$ of $D$ (line 35 ), and threshold decrypts $X_{\text {outgate }} \cdot D^{-1}$ to $y=r-d$ (line 37). Apart from this, the output phase is as in [CDN01]. Finally, in the proof phase, the simulator provides an honest proof to a corrupted verifier; or receives the proof of a corrupted result party for an honest verifier. In the latter

\footnotetext{
${ }^{5}$ Note that, instead of invoking $\Sigma_{\mathrm{PK}}$ 's simulator, it could equivalently have made a real proof; but in our security proof, we need that $\mathcal{S}_{\text {UVCDN }}^{\text {honest }}$ can function just using the encryption $X_{i}$, which is done using the simulator.

6 Technically, the multiplication simulator of [CDN01] uses an encryption of the result that it determined at the start of the CDN simulation, whereas we in effect determine this encryption during the gate-by-gate evaluation of the circuits. These two methods are clearly equivalent.
} 
case, if the verifier receives a correct proof, then the randomness used will be $s$ (as we will show later), so the simulator provides this randomness to the trusted party.

Lemma 2. For all inputs $x_{1}, \ldots, x_{m}, C, a$ with $|\mathcal{P} \cap C|<\lceil n / 2\rceil, \mathcal{S}_{U V C D N, \mathcal{A}}^{\text {honest }}$ runs in polynomial time, and

$$
\operatorname{EXE}_{U V C D N, \mathcal{A}}\left(k ; x_{1}, \ldots, x_{m} ; C ; a\right) \text { and } \operatorname{IDEA} L_{f, \mathcal{S}_{U V C D N, \mathcal{A}}}\left(k ; x_{1}, \ldots, x_{m} ; C ; a\right)
$$

are computationally indistinguishable in security parameter $k$.

Proof. Clearly, $\mathcal{S}_{\text {UVCDN }}^{\text {honest }}$ is p.p.t. since it only calls p.p.t. subroutines.

For computation indistinguishability, we extend the proof for the CDN protocol from [CDN01]. In this proof, an algorithm $\operatorname{YAD}_{\mathcal{A}}\left(B, k,\left(x_{1}, \ldots, x_{m}\right), C, a\right)$ is defined that, depending on an encrypted bit $B=(1+N)^{b} r^{N}$, simulates either an execution of the protocol with the real inputs (if $B$ encrypts 1 ); or an execution with zero inputs for the honest parties (if $B$ encrypts 0 ). It is shown that the $b=0$ case is statistically indistinguishable to $\operatorname{IDEAL}_{f, \mathcal{S}_{\mathcal{A}}}\left(k,\left(x_{1}, \ldots, x_{m}\right), C, a\right)$ and the $b=1$ case is statistically indistinguishable to $\operatorname{EXEC}_{\mathrm{CDN}, \mathcal{A}}\left(k,\left(x_{1}, \ldots, x_{m}\right), C, a\right)$. Moreover, the $b=0$ and $b=1$ cases are computationally indistinguishable by semantic security of the Paillier threshold cryptosystem (otherwise, the distinguisher between the two cases can distinguish random encryptions of 0 and 1).

To prove computational indistinguishability for the UVCDN protocol, we need to modify the algorithm $\operatorname{YAD}_{\mathcal{A}}\left(B, k,\left(x_{1}, \ldots, x_{m}\right), C, a\right)$ to take into account the changed output of the result party and the additional output of the verifier. Namely, for honest $\mathcal{R}, \mathrm{YAD}_{\mathcal{A}}$ outputs the result of the (real) computation and randomness $s$ for encryption $D$ from line 27 of the protocol; for honest $\mathcal{V}, \mathrm{YAD}_{\mathcal{A}}$ outputs the return value of vercomp. Statistical indistinguishability of the $b=1$ case to $\operatorname{EXEC}_{\mathrm{UVCDN}, \mathcal{A}}$ and computational indistinguishability of the $b=0$ and $b=1$ cases are proven analogously to [CDN01]. For statistical

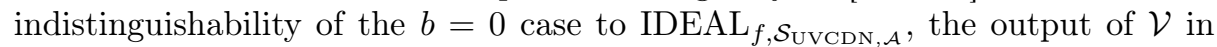
case of a corrupted $\mathcal{R}$ is problematic: in $\mathrm{YAD}_{\mathcal{A}}$ it returns the output of vercomp,

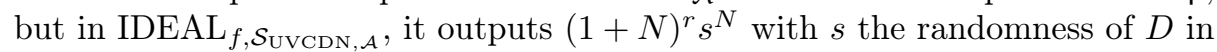
line 27 of UVCDN. These values coincide if $\mathcal{R}$ provides the same $Y_{0}$ and $D$ to $\mathcal{V}$ that it computed with the computation parties during the protocol; but $\mathcal{R}$ could of course present a $Y_{0}$ or $D$ with a valid proof to $\mathcal{V}$, in which case the output of $\mathcal{V}$ in the two distributions is different.

However, this latter possibility can only happen with negligible probability. For suppose that it happens with non-negligible probability. Because $Y_{0}$ and $D$ are both input to the proof of correct decryption ( $D$ being the auxiliary input), $\mathcal{R}$ needs to provide a valid proof of correct decryption apart from the one produced in line 31 of UVCDN. But from this proof, witness $\Delta^{2} d$ can be extracted by witness-extended emulation. From this, we can build a distinguisher that, given an encryption $B$, runs $\operatorname{YAD}_{\mathcal{A}}\left(r^{N}, k,\left(x_{1}, \ldots, x_{m}\right), C, a\right)$ ( $r$ random), and, whenever it gets witness $\Delta^{2} d$, outputs the decryption of $B$. By the above discussion, this distinguisher succeeds with non-negligible probability, contradicting semantic security of the Paillier threshold cryptosystem. So, IDEAL ${ }_{f, \mathcal{S}_{\mathrm{UVCDN}, \mathcal{A}}}$ and $\mathrm{YAD}_{\mathcal{A}}$ with $b=0$ are in fact statistically indistinguishable, as we needed to show. This completes the proof. 


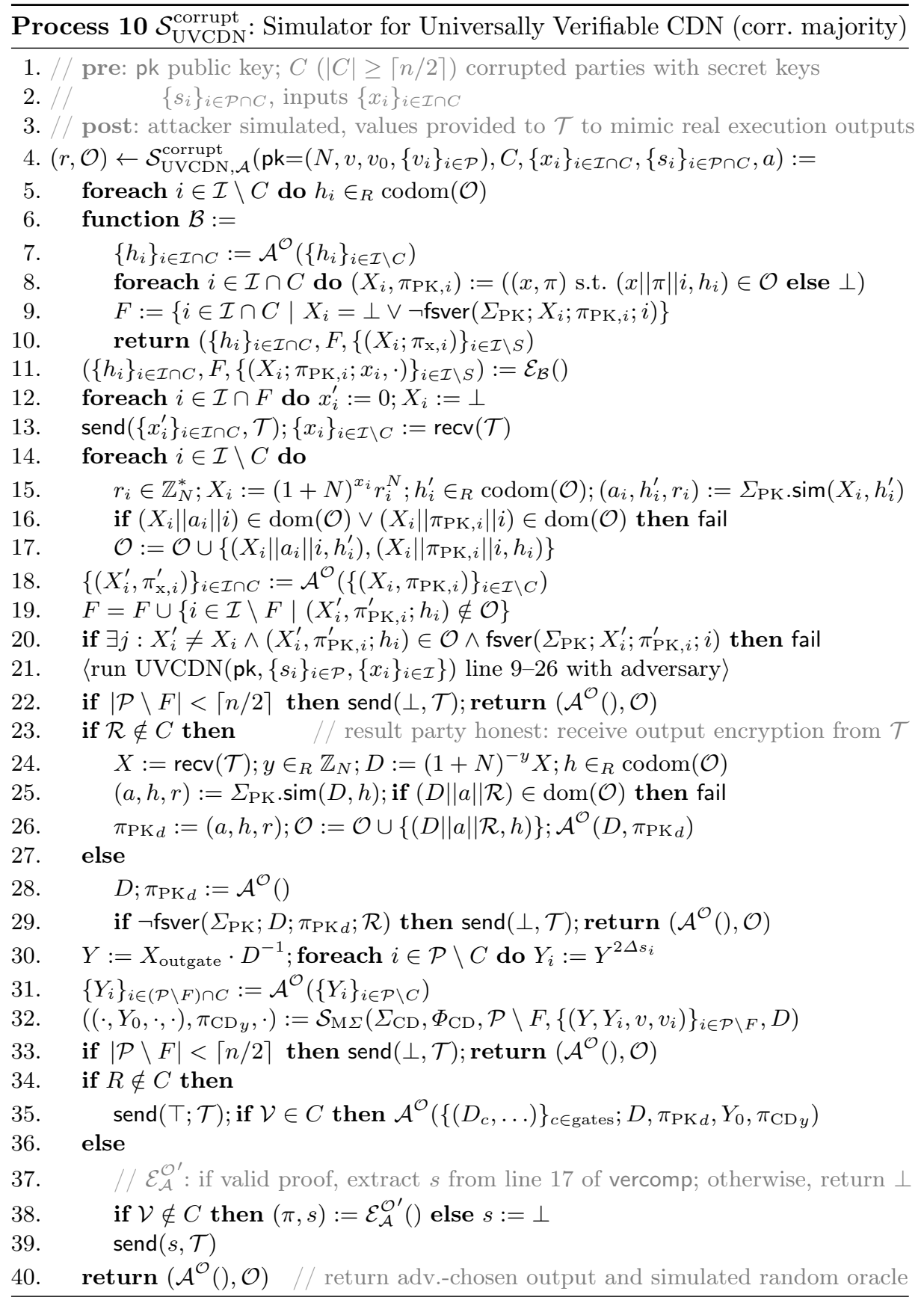

\section{C.2 The Corrupted Majority Case}

If at least $\lceil n / 2\rceil$ parties are corrupted, then the simulator has to simulate an adversary that can decrypt all values that it sees. In this case, $\mathcal{S}_{\text {UVCDN }}^{\text {corrupt }}$ (Pro- 
cess 10) performs a run of the actual protocol with respect to the adversary. It can do this, because it gets the inputs of the honest parties from $\mathcal{T}_{\mathrm{VSFE}}$ (line 6); and it can compute their shares of the decryption key by Lagrange interpolation from the shares of the corrupted parties ${ }^{7}$. There are two difficulties. The first is that, in the input phase, the simulator needs to provide the inputs of the corrupted parties to $\mathcal{T}_{\mathrm{VSFE}}$ before it receives the inputs of the honest parties. Hence, it needs to simulate the "hashed inputs" round of the UVCDN protocol without knowing the honest inputs. The second is that, in the output phase with a honest result party, the simulator obtains the encrypted output from $\mathcal{T}_{\text {VSFE }}$, and needs to force the computation to give this output.

In more detail, lines $5-20$ of $\mathcal{S}_{\text {UVCDN }}^{\text {corrupt simulate UVCDN's input phase. Recall }}$ that in UVCDN, the parties first provide hashes of their encrypted inputs, and then open these hashes to reveal the encryptions. When simulating the opening of these hashes, $\mathcal{S}_{\text {UVCDN }}^{\text {corrupt }}$ needs to provide actual encryptions of the honest parties' inputs: indeed, a corrupted majority can perform decryption so the encryptions need to be correct. Because $\mathcal{S}_{\text {UVCDN }}^{\text {corrupt }}$ needs to supply the corrupted inputs to $\mathcal{T}_{\text {VSFE }}$ before obtaining the honest inputs, it needs to extract the corrupted inputs already from the hashes. First, $\mathcal{S}_{\text {UVCDN }}^{\text {corrupt }}$ generates random hash images for the honest parties (line 5). Now, define a function $\mathcal{B}$ that exchanges the honest and corrupted hashes (line 7), inspects the oracle $\mathcal{O}$ to find the corrupted preimages (line 8), and checks their proofs (line 9 ). $\mathcal{B}$ is an algorithm that produces non-interactive zero-knowledge proofs of knowledge of the plaintexts of the corrupted $X_{i}$, so applying witness-extended emulation to $\mathcal{B}$ gives an algorithm that additionally gives the plaintexts $x_{i}$ (line 11 ). $\mathcal{S}_{\mathrm{UVCDN}}^{\text {corrupt }}$ supplies these inputs to $\mathcal{T}_{\text {VSFE }}$, and in return gets the inputs of the honest parties (line 13). Given the honest parties' inputs, $\mathcal{S}_{\text {UVCDN }}^{\text {corrupt }}$ can now compute real zero-knowledge proofs on behalf of the honest parties (line 15-16), and provide these to the adversary (line 18 ). Unless $\mathcal{S}_{\text {UVCDN }}^{\text {corrupt }}$ is unlucky with the random oracle (lines 16,20 ), this is a perfect simulation of the input phase with respect to the adversary.

In the computation phase, the simulator can do little else but perform the actual combination with the adversary (line 21): indeed, the adversary can decrypt all exchanged values so it can directly tell if the computation is manipulated.

In the result phase, if the result party is not corrupted, then $\mathcal{S}_{\text {UVCDN }}^{\text {corrupt }}$ receives the encrypted output $X$ of the computation (line 24). As in the honest majority case, it computes $D$ so that $X$ will indeed be output, and simulates $D$ 's proof (lines 25-26). Otherwise, the adversary chooses encryption $D$ (line 28). Finally, $\mathcal{S}_{\text {UVCDN }}^{\text {corrupt }}$ simulates the threshold decryption (line $30-32$ ). $\mathcal{S}_{\text {UVCDN }}^{\text {corrupt }}$ has now simulated the full protocol and just needs to check what its outcome was. If too many parties provided incorrect proofs, it signals to the trusted party that the computation was unsuccessful (line 33) If the result party is honest and the verifier is corrupted, then $\mathcal{S}_{\mathrm{UVCDN}}^{\text {corrupt }}$ simulates the provision of the proof to the adversary (line 35). Conversely, if the result party is corrupted and the verifier is not, then it receives the proof from the adversary. From this proof, it extracts

\footnotetext{
7 Actually, the simulator can only determine $\Delta s_{i}$ because it cannot divide by $\Delta$ under unknown modulus $p p^{\prime} q q^{\prime}$; but one observes that the UVCDN protocol always uses $s_{i}$ in combination with $\Delta$, so knowing $\Delta s_{i}$ is sufficient.
} 
the randomness of the encryption that is verified in line 17 of vercomp (line 38), and provides this randomness to the trusted party as the randomness of the result (line 39). (This encryption will typically be $D$ from line 28 , in which case the witness-extended emulator $\mathcal{E}_{\mathcal{A}}^{\mathcal{O}^{\prime}}$ implicitly rewinds the simulation.)

We achieve statistical indistinguishability (computational indistinguishability would be sufficient):

Lemma 3. For all inputs $x_{1}, \ldots, x_{m}, C, a$ with $|\mathcal{P} \cap C|>\lceil n / 2\rceil, \mathcal{S}_{U V C D N, \mathcal{A}}^{\text {corrupt }}$ runs in polynomial time, and

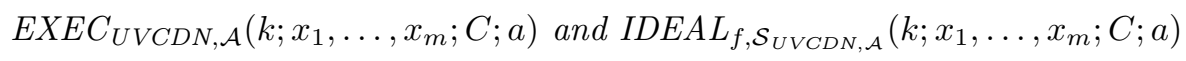

are statistically indistinguishable in security parameter $k$.

Proof. Clearly, $\mathcal{S}_{\mathrm{UVCDN}}^{\text {corrupt }}$ is p.p.t. since it only calls p.p.t. subroutines.

By construction, a full run of the protocol is simulated with respect to the adversary in a statistically indistinguishable way. It remains for us to check that also the output of honest $\mathcal{R}$ and $\mathcal{V}$ are indistinguishable.

In case the result party is corrupted, only the verifier produces an output. In the ideal world, a honest verifier outputs $(1+N)^{r} s^{N}$, where $r$ is the computation result; in the real world, a honest verifier outputs $(1+N)^{*} s^{N}$ for $D=(1+N)^{\dagger} s^{N}$ from line 21 of vercomp (or both output $\perp$ if the computation failed). Now, since $\mathcal{S}_{\text {UVCDN }}^{\text {corrupt }}$ does not simulate any proofs for statements that are not in the language of their respective $\Sigma$-protocols, and the adversary cannot produce any proofs for statements not in the language, with overwhelming probability all verifying proofs are for true statements, so also $(1+N)^{*} s^{N}$ has to be an encryption of the actual computation result. Hence we get indistinguishability.

Similarly, if the result party is honest, then the randomness it outputs in the ideal and real world is the same by construction; and the computation output has to be correct because the actual protocol was followed, and with overwhelming probability, no incorrect proofs have been produced. Hence, also in this case indistinguishability holds, which concludes the proof. 